\title{
Stability of quantum motion and correlation decay
}

\author{
Tomaž Prosen 团 and Marko Žnidarič [3] \\ Physics Department, Faculty of Mathematics and Physics, University of Ljubljana, \\ Jadranska 19, SI-1000 Ljubljana, Slovenia
}

\begin{abstract}
We derive a simple and general relation between the fidelity of quantum motion, characterizing the stability of quantum dynamics with respect to arbitrary static perturbation of the unitary evolution propagator, and the integrated time auto-correlation function of the generator of perturbation. Surprisingly, this relation predicts the slower decay of fidelity the faster decay of correlations is. In particular, for non-ergodic and non-mixing dynamics, where asymptotic decay of correlations is absent, a qualitatively different and faster decay of fidelity is predicted on a time scale $\propto 1 / \delta$ as opposed to mixing dynamics where the fidelity is found to decay exponentially on a time-scale $\propto 1 / \delta^{2}$, where $\delta$ is a strength of perturbation. A detailed discussion of a semi-classical regime of small effective values of Planck constant $\hbar$ is given where classical correlation functions can be used to predict quantum fidelity decay. Note that the correct and intuitively expected classical stability behavior is recovered in the classical limit $\hbar \rightarrow 0$, as the two limits $\delta \rightarrow 0$ and $\hbar \rightarrow 0$ do not commute. In addition we also discuss non-trivial dependence on the number of degrees of freedom. All the theoretical results are clearly demonstrated numerically on a celebrated example of a quantized kicked top.
\end{abstract}

To appear in J. Phys. A: Math. Gen. (March 2002)

PACS numbers: 03.65.Yz, 03.65.Sq, 05.45.Mt

\section{Introduction}

The precise quantum signatures of different qualitative types of classical motion and the very definition of chaos in quantum mechanics are still the issues of an unsettled discussion (see e.g. [1, 2, [3]). Due to unitarity of quantum dynamics, quantum chaos cannot be defined in the same way as the classical chaos [4], namely through the exponential sensitivity on the variation of initial conditions. However, Peres [5] proposed an alternative concept which can be used in classical as well as in quantum mechanics: One can study the stability of quantum motion with respect to a small variation in the Hamiltonian, or more generally, a variation of the unitary evolution operator. Clearly, in classical mechanics this concept, when applied to individual trajectories (or to phase space distribution functions as we show below in sect. 4), is equivalent to the sensitivity to initial conditions: Integrable systems with regular orbits are stable against small

$\ddagger$ prosen@fiz.uni-lj.si

$\S$ znidaricm@fiz.uni-lj.si 
variation in the hamiltonian (the statement of KAM theorem), wheres for chaotic orbits varying the hamiltonian has a similar effect as varying the initial condition: exponential divergence of two orbits for two nearby chaotic hamiltonians.

This paper is devoted to a systematic theoretical study of the stability of a unitary time evolution with respect to small static variations of the unitary propagator. It will be primarily applied to the Schrödinger propagator in quantum dynamics (sect. 2 and 3), however an alternative application to the stability of classical unitary PerronFrobenius evolution will be considered for comparison (sect. 4). The quantity of the central interest here is the fidelity of unitary (quantum) motion. Let us consider a unitary operator $U$ being either (i) a short-time propagator $U=\exp (-i H \Delta t / \hbar)$, or (ii) a Floquet map $U=\hat{\mathcal{T}} \exp \left(-i \int_{0}^{p} d \tau H(\tau) / \hbar\right)$ of (periodically time-dependent) Hamiltonian $H(H(\tau+p)=H(\tau))$, or (iii) a quantum Poincaré map or any other quantized symplectic map. In any case a general small perturbation of the unitary operator can be written in the following form

$$
U_{\delta}=U \exp (-i A \delta / \hbar)
$$

where $A$ is some self-adjoint operator, $\delta$ is a perturbation strength and $\hbar$ is an effective Planck constant which is taken out explicitly so that the observable $A$ has a well defined classical limit (e.g. the corresponding Weyl symbol). The influence of a small perturbation to the unitary evolution is quantitatively described by the overlap $\left\langle\psi_{\delta}(t) \mid \psi(t)\right\rangle$ measuring the Hilbert space distance between the exact and the perturbed time evolution from the same initial pure state $|\psi(t)\rangle=U^{t}|\psi\rangle,\left|\psi_{\delta}(t)\right\rangle=U_{\delta}^{t}|\psi\rangle$, where integer $t$ is a discrete time (in units of the period $p$ ). This defines the fidelity

$$
F(t)=\left\langle U_{\delta}^{-t} U^{t}\right\rangle
$$

where \langle\rangle gives the expectation value in the initial state $|\psi\rangle$. More generally, it may be useful to statistically average the fidelity over an ensemble of different pure initial states $\left|\psi_{k}\right\rangle$ appearing with probabilities $\rho_{k}$. Thus we will write the fidelity in terms of a statistical density operator $\rho=\sum_{k} \rho_{k}\left|\psi_{k}\right\rangle\left\langle\psi_{k}\right|$, namely as eq. (2) using the conventional statistical expectation value $\langle B\rangle=\operatorname{tr} \rho B$. The theoretical discussion in this paper is fully general, however, we will later apply our theory in the two extreme situations, namely for the (coherent) pure initial state $\rho=|\psi\rangle\langle\psi|$, and for the full Hilbert space average $\rho=\mathbb{1} / \mathcal{N}$ which is also equivalent to considering a random pure initial state of maximal information entropy. Integer $\mathcal{N}$ denotes a dimension of the relevant Hilbert space which can be written semiclassically by the Thomas-Fermi rule

$$
\mathcal{N}=(2 \pi \hbar)^{-d} \mathcal{V}
$$

where $\mathcal{V}$ is the classical phase-space (or energy shell) volume and $d$ is the number of (relevant) degrees of freedom.

The quantity $F(t)$, or its equivalent definitions, have already been discussed in several different contexts in quantum physics. We name just a few, namely those which provided direct motivation for the present work: First, $F(t)$ has been proposed by Peres [5] as a measure of the stability of quantum motion. Second, $|F(t)|^{2}$ is the Loschmidt 
echo characterizing the dynamical irreversibility, which has been used e.g. in spin-echo experiments [6] where one is interested in the overlap between the initial state $|\psi\rangle$ and an echo state $U_{\delta}^{-t} U^{t}|\psi\rangle$ obtained by composing forward time evolution, imperfect time inversion with a residual (non-invertible) interaction described by the operator $A \delta$, and backward time evolution. Third, the fidelity has become a standard measure characterizing the loss of phase coherence in the quantum computation [7]. Fourth, it was used to characterize the "hypersensitivity to perturbations" in related studies of information theoretic characterization of quantum chaos [8], though in a different context of a stochastically time-dependent perturbation.

The main result of this paper is a relation of the fidelity to the ergodic properties of quantum dynamics, more precisely to the time autocorrelation function of the generator of perturbation $A$. Quantum dynamics of finite and bound systems has always a discrete spectrum since the effective Hilbert space dimension $\mathcal{N}$ is finite, hence it is non-ergodic and non-mixing [9]: time correlation functions have fluctuating tails of order $\sim 1 / \mathcal{N}$. In order to reach a genuinely complex quantum motion with a continuous spectrum one has to enforce $\mathcal{N} \rightarrow \infty$ by considering one of the following two limits: semi-classical limit of effective Planck's constant $\hbar \rightarrow 0$, or thermodynamic limit of number of particles, or number of freedoms $d \rightarrow \infty$. Our result is surprising in the sense that it predicts the fidelity to decay slower if the integrated time correlation function is smaller, i.e. if the relaxation process is faster. In particular, for ergodic and sufficiently mixing dynamics (such that the time integrated autocorrelation function of the perturbation $A$ is finite) the fidelity is found to decay exponentially on a time-scale $\propto \delta^{-2}$, whereas for a 'more regular', non-ergodic dynamics with non-vanishing time-averaged correlation functions, the fidelity decay is found to be qualitatively faster with a characteristic timescale $\propto \delta^{-1}$. However, this surprising and apparently counterintuitive result is correctly reconciled with the expected corresponding behavior in the classical limit due to nontrivial non-interchangability of the limits $\delta \rightarrow 0$ and $\hbar \rightarrow 0$. In all cases, the relevant time-scale for the decay of fidelity in the semi-classical regime can be explicitly computed in terms of the classical quantities only (e.g. classical correlation functions). The main theoretical predictions are clearly demonstrated on the numerical examples of a kicked top and a pair of coupled kicked tops. Furthermore, our theory on fidelity is general and can be applied to any perturbed unitary evolution; as an example we consider the stability of the classical unitary Perron-Frobenius (Liouville) evolution for maps, where we show how 'classical fidelity' behaves in a qualitatively different way than the quantum fidelity and conforms to the corresponding linearly stable and exponentially unstable behaviors in the respective limiting cases of regular and chaotic dynamics. Two short announcements of our theory with applications in the contexts of many-body quantum dynamics and quantum computing have already been reported [10, 11].

In section 2, we present a theoretical derivation of the general relation between the fidelity of unitary motion and the correlation decay, discuss qualitatively different regimes of stability in the semiclassical range of small effective $\hbar$ and outline the corresponding time-scales. In section 3, our theoretical predictions are demonstrated 
in detail by numerical experiments on a quantized kicked top. In addition, we consider also a pair of coupled kicked tops in order to illustrate some dimensionality dependent aspects. In section 4, we demonstrate the conceptual difference between the stability of classical and quantum unitary evolution by applying our theory to the case of classical (Liouvillian) unitary evolution of phase space densities. In section 5, we conclude by pointing to some potentially important applications and implications of our results.

\section{General theory}

We start by rewriting the fidelity (2) in terms of the Heisenberg evolution of the perturbation $A_{t}:=U^{-t} A U^{t}$. Let us recursively insert the unity $U^{t^{\prime}} U^{-t^{\prime}}$ in the definition (2) for $F(t)$ and observe $U^{-t^{\prime}} U_{\delta}^{-1} U^{1+t^{\prime}}=\exp \left(i A_{t^{\prime}} \delta / \hbar\right)$ for $t^{\prime}$ running from $t-1$ downto 0 , yielding

$$
\begin{aligned}
F(t) & =\left\langle U_{\delta}^{-t} U^{t}\right\rangle=\left\langle U_{\delta}^{-(t-1)} U^{t-1} U^{-(t-1)}\left(U_{\delta}^{-1} U\right) U^{t-1}\right\rangle= \\
& =\left\langle U_{\delta}^{-(t-1)} U^{t-1} \exp \left(i A_{t-1} \delta / \hbar\right)\right\rangle= \\
& =\left\langle U_{\delta}^{-(t-2)} U^{t-2} \exp \left(i A_{t-2} \delta / \hbar\right) \exp \left(i A_{t-1} \delta / \hbar\right)\right\rangle= \\
& \cdots \\
& =\left\langle\exp \left(i A_{0} \delta / \hbar\right) \exp \left(i A_{1} \delta / \hbar\right) \cdots \exp \left(i A_{t-1} \delta / \hbar\right)\right\rangle .
\end{aligned}
$$

The obvious next step is to expand the product (4) into a power-series in $\delta$

$$
F(t)=1+\sum_{m=1}^{\infty} \frac{i^{m} \delta^{m}}{m ! \hbar^{m}} \hat{\mathcal{T}} \sum_{t_{1}, \ldots, t_{m}=0}^{t-1}\left\langle A_{t_{1}} A_{t_{2}} \cdots A_{t_{m}}\right\rangle,
$$

where the operator $\hat{\mathcal{T}}$ denotes a left-to-right time ordering. Such a perturbative expansion converges absolutely for any $\delta$ provided only that the perturbation $A$ is a bounded operator. Therefore, the fidelity may be computed by an arbitrary truncation of the above expansion (5) where its order $M(m \leq M)$ depends only on the desired accuracy $\mathcal{O}\left(\delta^{M+1}\right)$. We can see that the fidelity $F(t)$ can be expressed entirely in terms of time correlation functions of the generator $A$ of perturbation.

Before proceeding further we note that the first order term $(m=1)$ can be trivially eliminated, namely by transforming the perturbations as $A \rightarrow A-a \mathbb{1}$ with

$a:=(1 / t) \sum_{t^{\prime}=0}^{t-1}\left\langle A_{t^{\prime}}\right\rangle$ (i.e. the perturbation $A$ has to be traceless if $\rho=\mathbb{1} / \mathcal{N}$ ). This does not affect the absolute value $|F(t)|$ since a shift by a multiple of unity reflects in a simple complex rotation of fidelity $F(t) \rightarrow F(t) \exp (-i \delta a t / \hbar)$. So, to the lowest non-trivial order $(M=2)$ we find

$$
F(t)=1-\frac{\delta^{2}}{2 \hbar^{2}} \sum_{t^{\prime}, t^{\prime \prime}=0}^{t-1} \hat{\mathcal{T}} C\left(t^{\prime}, t^{\prime \prime}\right)+\mathcal{O}\left(\delta^{3}\right),
$$

where

$$
C\left(t^{\prime}, t^{\prime \prime}\right):=\left\langle A_{t^{\prime}} A_{t^{\prime \prime}}\right\rangle, \quad \hat{\mathcal{T}} C\left(t^{\prime}, t^{\prime \prime}\right)=C\left(\min \left\{t^{\prime}, t^{\prime \prime}\right\}, \max \left\{t^{\prime}, t^{\prime \prime}\right\}\right),
$$

is a 2-point time correlation function of the quantum observable $A$. In the following, the regime of sufficiently small $\delta$, such that the fidelity remains close to 1 (i.e. $1-F(t) \ll 1$ ) 
and the above formula (6) is accurate, will be referred to as the regime of linear response. Although we have a general convergent formula for systematic higher order corrections (5) we note that all the qualitative and in most cases even quantitative results of this paper can be explained by this simple linear-response Kubo-like formula (6).

An interesting and somewhat counterintuitive conclusion can be drawn from the expression (6), namely the smaller the time-integrated correlation function the higher the fidelity. In the semiclassical regime of approaching the classical limit $\hbar \rightarrow 0$ the quantum correlation function $C\left(t^{\prime}, t^{\prime \prime}\right)$ goes over to the corresponding classical correlation function provided a state $\rho$ converges to some classical phase space distribution, like e.g. for $\rho=\mathbb{1} / \mathcal{N}$.

Therefore, the fidelity for classically chaotic systems will decay with the rate which is inversely proportional to their rate of mixing, and furthermore for classically nonergodic, i.e. regular or integrable motion, the correlation functions will generally not decay to zero and the fidelity will therefore decay much faster. Of course, this surprising general conclusions can only be true if the initial state $|\psi\rangle$ (or the statistical density matrix $\rho$ ) is sufficiently random, i.e. if it is not a small linear combination of the eigenstates of $U\left(\right.$ or $\left.U_{\delta}\right)$ as will be discussed in more detail later. Now let us have a closer look at fidelity in the two qualitatively different regimes of correlation decay.

\subsection{Regime of ergodicity and fast mixing}

Here we assume that the system is (classically) ergodic and mixing such that the correlation function of the perturbation $A$ decays sufficiently fast; this typically (but not neccessarily!) corresponds to globally chaotic classical motion. Due to ergodicity we will safely assume $\rho=\mathbb{1} / \mathcal{N}$, i.e. average over the full Hilbert space $\langle\rangle=.\operatorname{tr}(.) / \mathcal{N}$. For any other initial state (e.g. in the worst case for the minimal wavepacket - coherent state) one obtains identical results on $F(t)$ for sufficiently long times, i.e. longer than the Ehrenfest - ergodic time $t_{\mathrm{E}} \approx \ln (1 / \hbar) / \lambda$ (for a classically chaotic system with maximal Lyapunov exponent $\lambda$ ) needed for a minimal wavepacket to spread effectively over the accessible phase space. The state averaged quantum correlation function is homogeneous in time $C\left(t^{\prime}, t^{\prime \prime}\right)=C\left(t^{\prime \prime}-t^{\prime}\right)$ so we simplify the second order - linear response - formula for the fidelity

$$
F(t)=1-\frac{\delta^{2}}{\hbar^{2}}\left\{\frac{1}{2} t C(0)+\sum_{t^{\prime}=1}^{t-1}\left(t-t^{\prime}\right) C\left(t^{\prime}\right)\right\}+\mathcal{O}\left(\delta^{3}\right),
$$

and note again that we have assumed $A$ to be traceless $\langle A\rangle=0$.

If the decay of correlation function $C(t)$ is sufficiently fast, namely it is sufficient that the sums $I_{k}=\sum_{t=-\infty}^{\infty}\left|t^{k} C(t)\right|$ exist for $k \in\{0,1\}$ and that a certain characteristic mixing time exists, e.g. $t_{\text {mix }}=I_{1} / I_{0}$, then the above formula can be further simplified. For times $t \gg t_{\text {mix }}$ we can neglect the second term under the summation in (\$) and

|| In case of autonomous (time-independent) systems, canonical or micro-canonial state should be used instead. 
obtain a linear decay in time $t$ in the linear response regime

$$
F_{\mathrm{em}}(t)=1-(\delta / \hbar)^{2} \sigma t \quad \text { with } \quad \sigma=\frac{1}{2} C(0)+\sum_{t=1}^{\infty} C(t)
$$

which is a transport coefficient given by the integral of correlation function. We can make a stronger statement in a non-linear-response regime if we make an additional assumption on the factorization of higher order time-correlations $-n$-point mixing. This implies that $2 m$-point correlation $\left\langle A_{t_{1}} \cdots A_{t_{2 m}}\right\rangle$ is appreciably different from zero for $t_{2 m}-t_{1} \rightarrow \infty$ only if all the (ordered) time indices $\left\{t_{j}, j=1 \ldots 2 m\right\}$ are paired with the time differences within each pair $t_{2 j}-t_{2 j-1}$ being of the order or less than $t_{\text {mix }}$. Then we can make a further reduction, namely if $t \gg m t_{\text {mix }}$

$$
\hat{\mathcal{T}} \sum_{t_{1}, \ldots, t_{2 m}=0}^{t-1}\left\langle A_{t_{1}} A_{t_{2}} \cdots A_{t_{2 m}}\right\rangle \rightarrow \hat{\mathcal{T}} \sum_{t_{1}, \ldots, t_{2 m}=0}^{t-1}\left\langle A_{t_{1}} A_{t_{2}}\right\rangle \cdots\left\langle A_{t_{2 m-1}} A_{t_{2 m}}\right\rangle \rightarrow \frac{(2 m) !}{m !}(t \sigma)^{m}
$$

so that we obtain a global exponential decay

$$
F_{\mathrm{em}}(t)=\exp \left(-t / \tau_{\mathrm{em}}\right), \quad \tau_{\mathrm{em}}=\frac{\hbar^{2}}{\delta^{2} \sigma},
$$

with a time-scale $\tau_{\mathrm{em}}=\mathcal{O}\left(\delta^{-2}\right)$. We should stress again that the above result (11) has been derived by the assumption of true quantum mixing which can be justified only in the limit $\mathcal{N} \rightarrow \infty$, e.g. either in semiclassical or thermodynamic limt. However, precise study of finite size effects and the relevant time and perturbation scales will be given in subsection 2.3.

\subsection{Non-mixing and non-ergodic regime}

The opposite situation of a non-mixing and non-ergodic quantum dynamics, which typically corresponds to integrable, quasi-integrable (KAM), or mixed classical dynamics, is characterized by a non-vanishing time-average of the correlation function

$$
\bar{C}=\lim _{t \rightarrow \infty} \frac{1}{t^{2}} \sum_{t^{\prime}, t^{\prime \prime}=0}^{t-1} \hat{\mathcal{T}} C\left(t^{\prime}, t^{\prime \prime}\right) .
$$

Here, due to non-ergodicity, time-average $\bar{C}$ depends on the choice of the initial state $|\psi\rangle$ or, more generally, on the density matrix $\rho$. We assume that a characteristic averaging time-scale $t_{\text {ave }}$ exists, namely it is an effective time $t=t_{\text {ave }}$ at which the limiting process (12) converges. Therefore, for sufficiently large times $t \gg t_{\text {ave }}$ the linear-response formula (6) gives, in contrast to (9), a simple quadratic decay in time

$$
F_{\text {ne }}(t)=1-\frac{1}{2}\left(\frac{t}{\tau_{\text {ne }}}\right)^{2}+\mathcal{O}\left(\delta^{3}\right), \quad \tau_{\text {ne }}=\frac{\hbar}{\delta \sqrt{\bar{C}}},
$$

with time-scale $\tau_{\text {ne }}=\mathcal{O}\left(\delta^{-1}\right)$. One should observe that the non-ergodic time-scale $\tau_{\text {ne }}$ can be much smaller than the ergodic-mixing time-scale $\tau_{\text {em }}$ (11) provided $\hbar$ is fixed, or the limit $\delta \rightarrow 0$ is taken prior to the limit $\hbar \rightarrow 0$. Again, we can make much stronger general statement using an additional assumption. Namely, if we assume that $t \gg t_{\text {ave }}$ 
then we can re-write the $m$-tuple sums in the series (5) in terms of a time average perturbation operator

$$
\bar{A}=\lim _{t \rightarrow \infty}(1 / t) \sum_{t^{\prime}=0}^{t-1} A_{t^{\prime}},
$$

namely

$$
F_{\mathrm{ne}}(t)=\sum_{m=0}^{\infty} \frac{(i \delta t)^{m}}{\hbar^{m} m !}\left\langle\bar{A}^{m}\right\rangle=\langle\exp (i t \bar{A} \delta / \hbar)\rangle .
$$

Note that $\bar{A}$ is by construction an integral of motion $[12],[U, \bar{A}] \equiv 0$, and reduces to a trivial multiple of identity (in fact, it vanishes with our choice of $\langle A\rangle=0$ ) in the ergodic case of previous subsection. Whereas in an ergodic and mixing case, $m$-th order term of (5) grows with time only as $\mathcal{O}\left(t^{m / 2}\right)$ (for even $m$ ) since it is dominated by pair time correlations, here in a non-ergodic case, the non-trivial time average operator $\bar{A}$ already gives the dominant effect, namely $\mathcal{O}\left(t^{m}\right)$ for $m$-th order term of (5), so the effect of pair time correlations can safely be neglected for sufficiently long times $\left(t \gg t_{\text {ave }}\right)$. Observe also that time averaged correlation is just a second moment of the time average operator

$$
\bar{C}=\left\langle\bar{A}^{2}\right\rangle .
$$

In the semiclassical regime, $\bar{C}$ goes to a purely classical, $\hbar$-independent quantity $\bar{C}_{\mathrm{cl}}$ only for such initial states $|\psi\rangle$, corresponding to $\hbar$-independent distribution of initial conditions in the classical phase space, e.g. for random states or $\rho=\mathbb{1} / \mathcal{N}$. Formula (15) expresses the fidelity in terms of a sequence of moments $\left\langle\bar{A}^{m}\right\rangle$. It has been shown [10] that for a general class of one-dimensional spin chains, the moments in thermodynamic limit $d \rightarrow \infty$ of long chains tend to a normal behavior $\left\langle\bar{A}^{2 m}\right\rangle \rightarrow(2 m-1) ! ! \bar{C}^{m}$, $\left\langle\bar{A}^{2 m+1}\right\rangle \rightarrow 0$, so fidelity exhibits a gaussian decay

$$
F_{\text {normal }}(t)=\exp \left(-\left(t / \tau_{\text {ne }}\right)^{2} / 2\right) \text {. }
$$

Although good numerical agreement with this formula has been observed in several related contexts, e.g. in quantum computing [11], it cannot be generally valid, in particular not in the semiclassical regime with a small number of freedoms $d$, as we show below.

In general, we can rewrite the formula (15) in terms of (average) local density of states (LDOS) of the time average $\bar{A}$ which is defined (for finite $\mathcal{N}$ ) as

$$
d_{\rho}(a)=\sum_{n} \delta\left(a-a_{n}\right)\left\langle a_{n}|\rho| a_{n}\right\rangle .
$$

where $a_{n}$ are the eigenvalues and $\left|a_{n}\right\rangle$ the eigenstates of self-adjont operator $\bar{A}$. Namely

$$
\langle\exp (i \delta \bar{A} t / \hbar)\rangle=\int d a e^{i a t \delta / \hbar} d_{\rho}(a),
$$

so the fidelity $F_{\text {ne }}(t)$ of non-ergodic systems is just a Fourier transformation of LDOS $d_{\rho}(a)$ of the time average perturbation $\bar{A}$ (which becomes the simple (global) density of states in case of an uniform average $\rho=\mathbb{1} / \mathcal{N})$. Thus only a gaussian LDOS would yield a gaussian fidelity decay (17), however we often find good finite time gaussian behavior 
which goes beyond the second moment $\int d a a^{2} d_{\rho}(a)=\left\langle\bar{A}^{2}\right\rangle=\bar{C}$, even if the asymptotic time dependence is completely different, see e.g. fig. 7 for an example of kicked top.

Let us now try to give some semi-classical estimates on the fidelity decay in nonergodic regime by expressing the asymptotics $\hbar \rightarrow 0$ in terms of classical information only. Here we assume that our (classical) map is integrable so that there exist $d$ canonical constants of motion - the action variables $\boldsymbol{I}=\left(I_{1}, \ldots, I_{d}\right)$, which are quantized using EBK rule $\boldsymbol{I}_{\boldsymbol{n}}=\hbar(\boldsymbol{n}+\boldsymbol{\gamma} / 4)$ where integers $n_{j} \geq 0, j \in\{1, \ldots, d\}$ are quantum numbers and integers $\gamma_{j}$ Maslov indices. Since the time average operator $\bar{A}$ commutes with $U$ and with the actions $\boldsymbol{I}$, it is diagonal in (generically non-degenerate) basis of eigenstates of $\boldsymbol{I}$ (quantized tori) $|\boldsymbol{n}\rangle$, namely in the leading semiclassical order

$$
\left\langle\boldsymbol{n}|\bar{A}| \boldsymbol{n}^{\prime}\right\rangle=\delta_{\boldsymbol{n}, \boldsymbol{n}^{\prime}} \bar{a}\left(\boldsymbol{I}_{\boldsymbol{n}}\right)
$$

where $\bar{a}(\boldsymbol{I})$ is the corresponding classical time-averaged observable in action space. The fidelity (15) can therefore be written as

$$
F_{\text {ne }}(t)=\sum_{\boldsymbol{n}} \exp \left(i t \bar{a}\left(\boldsymbol{I}_{\boldsymbol{n}}\right) \delta / \hbar\right)\langle\boldsymbol{n}|\rho| \boldsymbol{n}\rangle
$$

Now, provided the diagonal elements of the density matrix can be written in terms of some smooth function $D_{\rho}\left(\boldsymbol{I}_{\boldsymbol{n}}\right)=\langle\boldsymbol{n}|\rho| \boldsymbol{n}\rangle$, and replacing the sum (21) by an integral over the action space, which is justified for small $\hbar$ up to classically long time $\propto \hbar^{0} \delta^{-1}$, we obtain

$$
F_{\text {ne }}(t)=\hbar^{-d} \int d^{d} \boldsymbol{I} \exp \{i t \bar{a}(\boldsymbol{I}) \delta / \hbar\} D_{\rho}(\boldsymbol{I})
$$

The obvious next step is to compute this integral by a method of stationary phase. However, the result depends on the precise form of the function $D_{\rho}(\boldsymbol{I})$ (which may also explicitly depend on $\hbar$ ) so we only work out the details for two important special cases.

2.2.1. Semiclassical asymptotics for an uniform initial state average. Let us first assume uniform averaging over (random) initial states $\rho=\mathbb{1} / \mathcal{N}$, so $D_{\rho}(\boldsymbol{I}) \equiv 1 / \mathcal{N}=$ $(2 \pi \hbar)^{d} / \mathcal{V}$. Then, for large $t \delta / \hbar$ the above integral (22) can be written as a sum of contributions stemming from, say $p$ points, $\boldsymbol{I}_{\eta}, \eta=1, \ldots, p$ where the phase is stationary, $\partial \bar{a}\left(\boldsymbol{I}_{\eta}\right) / \partial \boldsymbol{I}=0$, yielding

$$
F_{\text {ne }}^{\text {ave }}(t)=\frac{(2 \pi)^{3 d / 2}}{\mathcal{V}}\left|\frac{\hbar}{t \delta}\right|^{d / 2} \sum_{\eta=1}^{p} \frac{\exp \left\{i t \bar{a}\left(\boldsymbol{I}_{\eta}\right) \delta / \hbar+i \nu_{\eta}\right\}}{\left|\operatorname{det} \overline{\mathbf{A}}_{\eta}\right|^{1 / 2}},
$$

where $\left\{\overline{\mathbf{A}}_{\eta}\right\}_{j k}:=\partial^{2} \bar{a}\left(\boldsymbol{I}_{\eta}\right) / \partial I_{j} \partial I_{k}$ is a matrix of second derivatives at the stationary point $\eta$, and $\nu_{\eta}=\pi\left(m_{+}-m_{-}\right) / 4$ where $m_{ \pm}$are the numbers of positive/negative eigenvalues of the matrix $\overline{\mathbf{A}}_{\eta}$. We repeat that the stationary phase formula (23) is expected to be correct in the range const $\hbar / \delta<t<\operatorname{const}^{\prime} / \delta$. Most interesting to note is the asymptotic power-law time and perturbation dependence $F_{\text {ne }}^{\text {ave }} \sim|\hbar /(t \delta)|^{d / 2}$, which allows for a possible crossover to a gaussian decay (17) when approaching the thermodynamic limit $d \rightarrow \infty$. 
2.2.2. Semiclassical asymptotics for a coherent initial state. Now let us consider a single $d$-dimensional general coherent state centered at $\left(\boldsymbol{I}^{*}, \boldsymbol{\theta}^{*}\right)$ in action-angle space, $\rho=\left|\boldsymbol{I}^{*}, \boldsymbol{\theta}^{*}\right\rangle\left\langle\boldsymbol{I}^{*}, \boldsymbol{\theta}^{*}\right|$,

$$
\left\langle\boldsymbol{n} \mid \boldsymbol{I}^{*}, \boldsymbol{\theta}^{*}\right\rangle=\left(\frac{\hbar}{\pi}\right)^{d / 4}|\operatorname{det} \Lambda|^{1 / 4} \exp \left\{-\frac{1}{2 \hbar}\left(\boldsymbol{I}_{\boldsymbol{n}}-\boldsymbol{I}^{*}\right) \cdot \Lambda\left(\boldsymbol{I}_{\boldsymbol{n}}-\boldsymbol{I}^{*}\right)-i \boldsymbol{n} \cdot \boldsymbol{\theta}^{*}\right\},
$$

where $\Lambda$ is a positive symmetric $d \times d$ matrix of squeezing parameters, giving $D_{\rho}(\boldsymbol{I})=$ $(\hbar / \pi)^{d / 2}|\operatorname{det} \Lambda|^{1 / 2} \exp \left(-\left(\boldsymbol{I}-\boldsymbol{I}^{*}\right) \cdot \Lambda\left(\boldsymbol{I}-\boldsymbol{I}^{*}\right) / \hbar\right)$ and

$$
F_{\mathrm{ne}}^{\mathrm{coh}}(t)=\frac{|\operatorname{det} \Lambda|^{1 / 2}}{(\pi \hbar)^{d / 2}} \int d^{d} \boldsymbol{I} \exp \left\{\frac{1}{\hbar}\left(i t \bar{a}(\boldsymbol{I}) \delta-\left(\boldsymbol{I}-\boldsymbol{I}^{*}\right) \cdot \Lambda\left(\boldsymbol{I}-\boldsymbol{I}^{*}\right)\right)\right\} .
$$

Using the assumption $\delta t \ll 1$, we see that a unique stationary point $\boldsymbol{I}_{s}$ of the exponent approaches $\boldsymbol{I}^{*}$ as $\delta \rightarrow 0$,

$$
\boldsymbol{I}_{s}=\boldsymbol{I}^{*}-\frac{i t \delta}{2} \Lambda^{-1} \boldsymbol{a}^{\prime}+\mathcal{O}\left(\delta^{2}\right), \quad \text { where } \quad \boldsymbol{a}^{\prime}:=\frac{\partial \bar{a}\left(\boldsymbol{I}^{*}\right)}{\partial \boldsymbol{I}}
$$

so we may explicitly evaluate (25) by the method of stationary phase without any lower bound on the range of time $t$,

$$
F_{\mathrm{ne}}^{\mathrm{coh}}(t)=\exp \left\{-\frac{\left(\boldsymbol{a}^{\prime} \cdot \Lambda^{-1} \boldsymbol{a}^{\prime}\right) \delta^{2}}{4 \hbar} t^{2}+\frac{i \bar{a}\left(\boldsymbol{I}^{*}\right) \delta}{\hbar} t\right\} .
$$

Note that the fidelity decay for a coherent initial state with regular classical motion has a time-scale

$$
\tau_{\text {ne-coh }}=(2 \hbar)^{1 / 2}\left(\boldsymbol{a}^{\prime} \cdot \Lambda^{-1} \boldsymbol{a}^{\prime}\right)^{-1 / 2} \delta^{-1} \propto \hbar^{1 / 2} \delta^{-1}
$$

which is consistent with (13) with $\bar{C}=\frac{1}{2} \hbar\left(\boldsymbol{a}^{\prime} \cdot \Lambda^{-1} \boldsymbol{a}^{\prime}\right)$ and is typically (for small $\hbar$ ) larger than the time-scale $\tau_{\text {ne }} \propto \hbar / \delta$ for a random initial state. However, surprisingly enough, for sufficiently small $\delta$, fidelity decay for coherent state with regular classical dynamics can still be faster than the fidelity decay for ergodic and mixing dynamics. It should be noted that the above derivation for fidelity decay of a coherent state (2428) remains valid in a quasi-integrable (KAM) situation of mixed classical phase space, provided that the initial wave packet is launched in a regular region of phase space where (local) action-angle variables exist.

From the derivation of gaussian behavior (27) we can also begin to understand the reason for a faster gaussian decay of fidelity for a non-mixing system in contrast to the exponential decay for a mixing case. Namely, in the non-mixing case, the displacement between centers of the wave packets of two nearby regular evolutions grows ballistically and thus produces destructive interference (i.e. decay of fidelity) much faster than in the case of mixing dynamics where the destructive interference builds up in a diffusive, random way.

\subsection{Finite size effects and time and perturbation scales}

The theoretical relations of the previous subsections are strictly justified in the asymptotics $\mathcal{N} \rightarrow \infty$. However, in any experimentally or practically relevant situation 
the dimensionality of the relevant Hilbert (sub)space $\mathcal{N}$ is finite, so we have to discuss in detail the limitations and additional time and perturbation scales associated with this.

First of all, for finite $\mathcal{N}$, fidelity $F(t)$ cannot decay indefinitely but starts to fluctuate for long times due to discreteness of the spectrum of the evolution operator $U$. Let us write the eigenphases of $U$ and $U_{\delta}$, and the corresponding eigenvectors, respectively, as $\phi_{n}, \phi_{n}^{\delta}$, and $\left|\phi_{n}\right\rangle,\left|\phi_{n}^{\delta}\right\rangle, n=1, \ldots, \mathcal{N}$, satisfying $U\left|\phi_{n}\right\rangle=e^{-i \phi_{n}}\left|\phi_{n}\right\rangle, U_{\delta}\left|\phi_{n}^{\delta}\right\rangle=e^{-i \phi_{n}^{\delta}}\left|\phi_{n}^{\delta}\right\rangle$. Let us define a unitary operator $V$ which maps the eigenbasis of $U$ to the eigenbasis of $U_{\delta}$, namely $V\left|\phi_{n}\right\rangle:=\left|\phi_{n}^{\delta}\right\rangle$ for all $n$, with matrix elements $V_{m n}:=\left\langle\phi_{m}|V| \phi_{n}\right\rangle$, and write the matrix elements of the statistical averaging operator as $\rho_{m n}:=\left\langle\phi_{m}|\rho| \phi_{n}\right\rangle$. Note that the matrix $V_{m n}$ is real orthogonal, $V \in O(\mathcal{N})$, if $U$ and $U_{\delta}$ possess a common anti-unitary symmetry (e.g. time-reversal). Now it is straightforward to rewrite the fidelity (2) as

$$
F(t)=\sum_{n, l}(\rho V)_{n l} V_{n l}^{*} \exp \left(i\left(\phi_{n}-\phi_{l}^{\delta}\right) t\right),
$$

where $(\rho V)_{n l}=\sum_{m} \rho_{n m} V_{m l}$ is a matrix element of the operator product $\rho V$. At this point we are interested in the long time fluctuations so we compute the time average fidelity fluctuation

$$
F_{\mathrm{rms}}^{2}:=\overline{|F|^{2}}=\sum_{n, l}\left|(\rho V)_{n l}\right|^{2}\left|V_{n l}\right|^{2} .
$$

In the process of averaging over the time we have assumed that the eigenphases are non-degenerate so $\overline{\exp \left(i\left(\phi_{n}-\phi_{n^{\prime}}+\phi_{l}^{\delta}-\phi_{l^{\prime}}^{\delta}\right) t\right)}=\delta_{n n^{\prime}} \delta_{l, l^{\prime}}$. So we see that fidelity fluctuation $F_{\text {rms }}$ depends on the orthogonal/unitary matrix $V_{m n}$ and the statistical matrix $\rho_{m n}$. Clearly, $V_{m n}$ converges to an identity matrix for sufficiently small $\delta$, while for large enough $\delta$ and sufficiently complex perturbation $A$ one may assume that it becomes a random orthogonal/unitary matrix. Of course, the critical perturbation strength $\delta=\delta_{\text {rm }}$ for a transition between the two regimes is very much system dependent and cannot be discussed in general. Let us now discuss three different limiting cases of the statistical matrix $\rho_{m n}$ :

(i) First, consider the simplest case where the initial state is a pure eigenstate of $U$ say, $\rho=\left|\phi_{1}\right\rangle\left\langle\phi_{1}\right|$. Then $\rho_{m n}=\delta_{m, 1} \delta_{n, 1}$ and (30) rewrites to

$$
F_{\text {rms-i }}^{2}=\sum_{l}\left|V_{1 l}\right|^{4} \text {. }
$$

Now, for weak perturbation $\delta \ll \delta_{\text {rm }}$ fidelity does not decay at all $F_{\text {rms }-\mathrm{i}}^{\text {weak }}=1$, while for strong perturbation $\delta \gg \delta_{\mathrm{rm}}$ we may estimate (31) using the fact that $V_{m n}$ become real/complex gaussian random variables with variance $1 / \mathcal{N}$ for the $\operatorname{orthogonal}(\beta=$ $1) /$ unitary $(\beta=2)$ case, namely $F_{\text {rms-i }}^{\text {strong }}=[(4-\beta) / \mathcal{N}]^{1 / 2}$. This result can be generalized to the case where initial state can be written as a finite superposition of a small number, say $K$, of eigenstates of $U$ : then for a weak perturbation $F_{\text {rms }}^{\text {weak }} \sim K^{-1 / 2}$. With this simple observation we can easily explain the numerical result of Peres [5] where 
no-decay of fidelity was found for a coherent initial state sitting in the center of elliptic island, thus being a superposition of a very small number of eigenstates (it is almost an eigenstate), while the behavior in generic cases may be drastically different as described in the present work.

(ii) Second, consider the case of a random pure initial state $|\psi\rangle=\sum_{n} c_{n}\left|\phi_{n}\right\rangle$, giving $\rho_{m n}=c_{m} c_{n}^{*}$. The principle of maximal entropy forces the coefficients $c_{n}$ to be independent random complex gaussian variables with variance $1 / \mathcal{N}$. Thus, averaging (30) over $c_{n}$ 's yields

$$
F_{\text {rms-ii }}^{2}=\frac{1}{\mathcal{N}^{2}}\left(\sum_{n, l}\left|V_{n l}\right|^{4}+\sum_{n, m, l}\left|V_{n l}\right|^{2}\left|V_{m l}\right|^{2}\right) .
$$

Here, for weak perturbation $\delta \ll \delta_{\text {rm }}$ we find $F_{\text {rms-ii }}^{\text {weak }}=(2 / \mathcal{N})^{1 / 2}$ while for strong perturbation $\delta \gg \delta_{\text {rm }}$ we have $F_{\text {rms-ii }}^{\text {strong }}=\mathcal{N}^{-1 / 2}$

(iii) Third, in the limiting case of a uniform average over all initial states $\rho=\mathbb{1} / \mathcal{N}$, $\rho_{m n}=\delta_{m n} / \mathcal{N}$, we have

$$
F_{\text {rms-iii }}^{2}=\frac{1}{\mathcal{N}^{2}} \sum_{n, l}\left|V_{n l}\right|^{4} .
$$

Again, for weak perturbation $\delta \ll \delta_{\text {rm }}, F_{\text {rms-iii }}^{\text {weak }}=\mathcal{N}^{-1 / 2}$, and for strong perturbation $\delta \gg \delta_{\text {rm }}, F_{\text {rms-iii }}^{\text {strong }}=(4-\beta)^{1 / 2} / \mathcal{N}$.

Note that the formulae (31-33) state simply that fidelity fluctuation is an inverse participation ratio (IPR) of the perturbed eigenstate(s) (single one in case (i) or an average over all in case (iii)) in terms of the unperturbed eigenstates, and is thus directly related to the localization properties of eigenstates of $U_{\delta}$ in terms of eigenstates of $U$. However, except for the pathological case of initial state being a small combination of eigenstates of $U$ with weak perturbation, the fidelity fluctuation is always between the limiting values $\mathcal{N}^{-1 / 2}$, and $\sqrt{3} / \mathcal{N}$. For an illustrations of transitions between weak and strong perturbations in case (iii) of a kicked top, see fig. 1.

Therefore, fidelity will decay according to one of the asymptotic formulae $(11,17,23,27)$ until it reaches the value of finite size fluctuation. This condition determines the time-scale $t_{*}=t_{*}(\mathcal{N})$,

$$
F_{\mathcal{N} \rightarrow \infty}\left(t_{*}(\mathcal{N})\right)=F_{\text {rms }}(\mathcal{N}) .
$$

In non-ergodic and non-mixing regime this is the only relevant finite size time-scale, while in the mixing regime the situation is more complicated.

2.3.1. Regime of ergodicity and fast mixing. Here, combining (34) with an exponential decay (11) we get

$$
t_{*}=\mu \tau_{\mathrm{em}} \ln \mathcal{N} \approx \frac{\mu \hbar^{2} d}{\delta^{2} \sigma_{\mathrm{cl}}} \ln (1 / \hbar)
$$




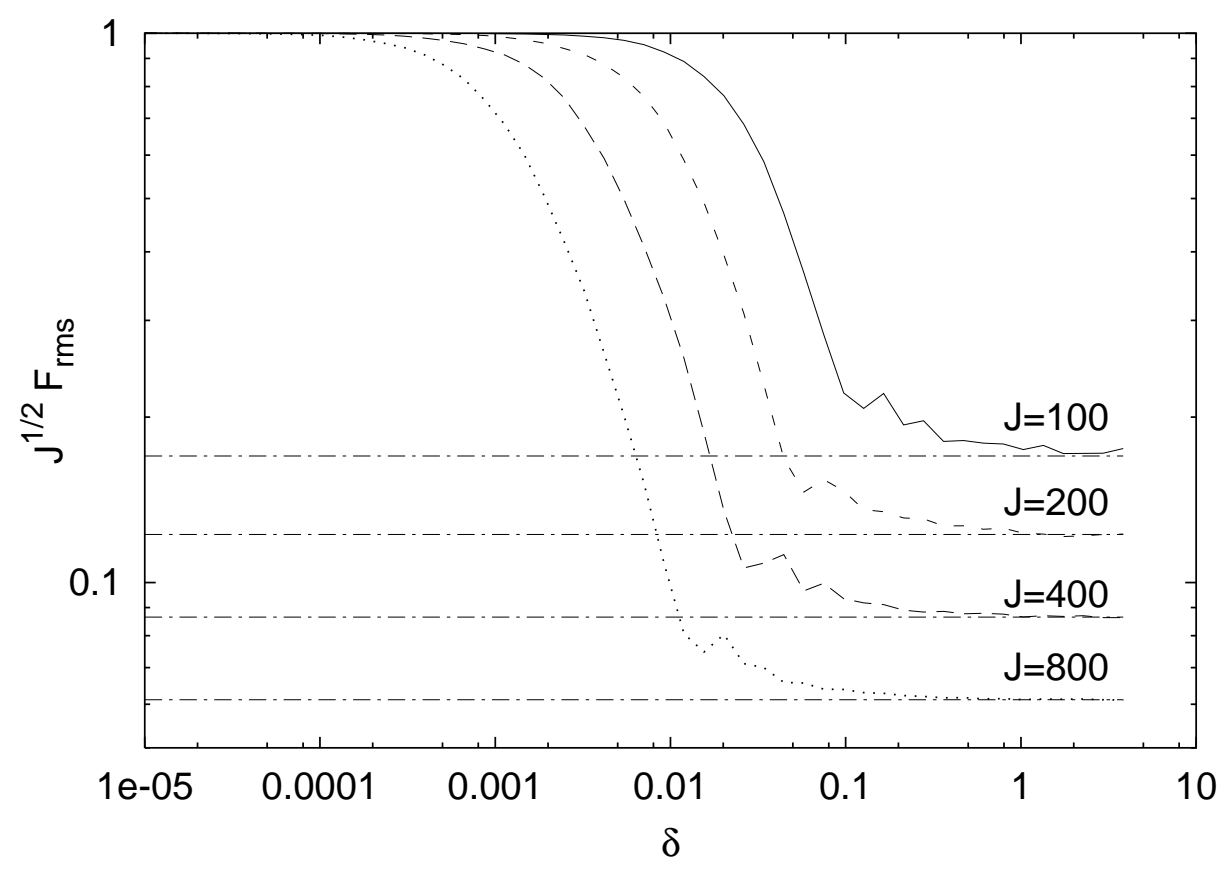

Figure 1. Finite-size fidelity fluctuation for a kicked top (45) in the mixing regime $(\alpha=30)$ and with the whole Hilbert space average (33) is shown as a function of the perturbation strength $\delta$ at four different values of $J(=1 / \hbar)=100,200,400,800$ (curves from right to left, respectively). Data are scaled in such a way $J^{1 / 2} F_{\text {rms }}$ (note that $\mathcal{N}=J$ ) that the weak perturbation regime corresponds to value 1 while the horizontal lines give the corresponding values in the strong perturbation regime $F_{\text {rms-iii }}^{\text {strong }}=\sqrt{3} / J$. Note that the crossover perturbation scales as $\delta_{\mathrm{rm}} \propto 1 / J$.

where $\mu=-\ln F_{\text {rms }} / \ln \mathcal{N}$ is a parameter which typically lies in the range $1 / 2<\mu<1$ (except if the initial state is non-random and the dynamics is non-ergodic (e.g. case (i)) and the perturbation is small $\delta \ll \delta_{\mathrm{rm}}$ ) and depends on IPR and the statistical operator $\rho$ as discussed above, and $\sigma_{\mathrm{cl}}$ is the classical limit of the transport coefficient (9).

The second new time-scale is related the to asymptotic non-decay of time correlations for finite $\mathcal{N}$ quantum dynamics, namely even if the system is classically mixing the quantum correlation function will have a small non-vanishing ( $\hbar$-dependent) time average

$$
\bar{C}=\overline{\left\langle A(t) A\left(t^{\prime}\right)\right\rangle}=\sum_{n} \rho_{n n} A_{n n}^{2}
$$

where $A_{m n}=\left\langle\phi_{m}|A| \phi_{n}\right\rangle$. However, since the classical system is ergodic and mixing, we will use a version of quantum chaos conjecture saying that [13] then $A_{m n}$ are independent gaussian random variables with a variance given by the Fourier transformation $S(\omega)$ of the corresponding classical correlation function $C_{\mathrm{cl}}(t)$ at frequency $\omega=\phi_{m}-\phi_{n}$. On the diagonal we have $\omega=0$ and additional factor of 2 due to random matrix measure on the diagonal (see e.g. [1]), so we can write

$$
\bar{C}=\frac{4 \sigma_{\mathrm{cl}}}{\mathcal{N}},
$$


where $\sigma_{\mathrm{cl}}=S(0) / 2$ is the classical limit of (9). Due to ergodicity, for large $\mathcal{N}$, this does not depend on the statistical operator $\rho$; neither in cases (ii,iii), nor in case (i) if one assumes additional ensemble averaging. The decay of fidelity (8) will start to be dominated by the average plateau (37) at time $t$, when $\sum_{t^{\prime}=0}^{t-1}\left(t-t^{\prime}\right) \bar{C} \approx 2 \sigma_{\mathrm{cl}} t^{2} / \mathcal{N} \geq \sigma_{\mathrm{cl}} t$, i.e. for times $t$ greater than $t_{\mathrm{p}}$

$$
t_{\mathrm{p}}=\frac{1}{2} \mathcal{N} \propto \hbar^{-d}
$$

which is just the Heisenberg time associated to the inverse density of states.

Now, depending on the interrelation among four (or five) time-scales $\tau_{\mathrm{em}} \propto \hbar^{2} \delta^{-2}$, $t_{\mathrm{p}} \propto \hbar^{-d} \delta^{0}, t_{*} \propto \hbar^{2} \ln (1 / \hbar) \delta^{-2} d, t_{\text {mix }} \propto \hbar^{0} \delta^{0}$, (and $t_{\mathrm{E}} \propto \ln (1 / \hbar) \delta^{0}$ if we are considering coherent initial states, like e.g. [14, 15, 16]) we can have four (or five) different regimes depending on three main scaling parameters: perturbation strength $\delta$, Planck's constant $\hbar$, and dimensionality $d$. Note that we always have $t_{*}>\tau_{\mathrm{em}}$. All different regimes can be reached by changing only $\delta$ while keeping $\hbar$ and $d$ fixed (see fig. (2):

(a) For sufficiently small perturbation $\delta$ we will have $t_{\mathrm{p}}<\tau_{\mathrm{em}}$. This means that $F_{\mathrm{em}}\left(t_{\mathrm{p}}\right) \approx 1$ and we will have initially quadratic decay (13) with $\bar{C}$ given by an average finite size plateau (37). This will occur for $\delta \leq \delta_{\mathrm{p}}$ where

$$
\delta_{\mathrm{p}}=\hbar\left(\frac{2}{\sigma_{\mathrm{cl}} \mathcal{N}}\right)^{1 / 2}=\frac{\sqrt{2}(2 \pi)^{d / 2}}{\left(\mathcal{V} \sigma_{\mathrm{cl}}\right)^{1 / 2}} \hbar^{d / 2+1} .
$$

In fact, in this regime, also referred to [16, 17] as perturbative, one may use a first order stationary perturbation theory on the eigenstates of $U_{\delta}$, yielding $\phi_{n}^{\delta}=\phi_{n}+A_{n n} \delta / \hbar+$ $\mathcal{O}\left(\delta^{2}\right), V_{m n}=\delta_{m n}+\mathcal{O}(\delta)$, and rewrite (following [17]) the finite size fidelity (29) in terms of a Fourier transform of a probability distribution $w(A)$ of diagonal matrix elements $A_{n n}, F_{\mathrm{p}}(t)=\int d A w(A) \exp (-i A t \delta / \hbar)$. Since $w(A)$ is conjectured to be gaussian for classically ergodic and mixing system [13], it follows that $F_{\mathrm{p}}(t)$ is also a gaussian with a semiclassically long time-scale $\tau_{\mathrm{p}}$

$$
F_{\mathrm{p}}(t)=\exp \left(-\left(t / \tau_{\mathrm{p}}\right)^{2} / 2\right), \quad \tau_{\mathrm{p}}=\left(\frac{\mathcal{N}}{\sigma_{\mathrm{cl}}}\right)^{1 / 2} \frac{\hbar}{2 \delta}=\frac{\mathcal{V}^{1 / 2}}{(2 \pi)^{d / 2} \sigma_{\mathrm{cl}}^{1 / 2}} \frac{\hbar^{1-d / 2}}{2 \delta} .
$$

(b) If $\tau_{\mathrm{em}}<t_{\mathrm{p}}<t_{*}$ we will have a crossover from initial exponential decay of fidelity (11) to a gaussian decay (40) at $t \sim t_{\mathrm{p}}$, which will terminate and go over to fluctuating behavior when $F_{\mathrm{p}}(t)=F_{\mathrm{rms}}(\mathcal{N})$. Note that this will happen before time $t_{*}$ which is estimated (34) based on a slower exponential decay (11). This regime will exist in perturbation range $\delta_{\mathrm{p}}<\delta<\delta_{\mathrm{s}}$ with an upper border $\delta_{\mathrm{s}}$ determined by the condition $t_{\mathrm{p}}=t_{*}$

$$
\delta_{\mathrm{s}}=(\mu \ln \mathcal{N})^{1 / 2} \delta_{\mathrm{p}} .
$$

(c) If we still further increase $\delta$, we have the most interesting, 'fully nonperturbative' regime, when $\tau_{\mathrm{em}}<t_{*}<t_{\mathrm{p}}$ and we will have a full exponential decay (11), up to time 


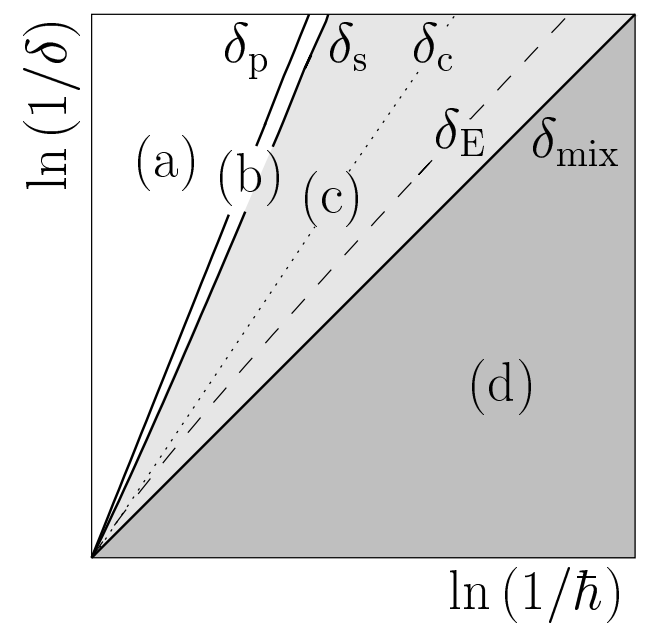

Figure 2. Schematic view of different regimes (a-d) of fidelity decay.

$t_{*}$ when the fidelity reaches finite size fluctuations. This regime continues for $\delta<\delta_{\text {mix }}$ where the border

$$
\delta_{\text {mix }}=\frac{\hbar}{\sqrt{\sigma_{\mathrm{cl}} t_{\mathrm{mix}}}}=\left(\frac{\mathcal{N}}{2 t_{\text {mix }}}\right)^{1 / 2} \delta_{\mathrm{p}} .
$$

is determined by the condition $\tau_{\mathrm{em}}=t_{\text {mix }}$ which is a point where the arguments leading to the factorization (10) and exponential decay (111) are no longer valid. We note that the relative size of this window range $\delta_{\text {mix }} / \delta_{\mathrm{s}}=\sqrt{\mathcal{N} /\left(t_{\text {mix }} \ln \mathcal{N}\right)}$ increases both, in the semiclassical and in the thermodynamic limit. This regime also corresponds to 'Fermi golden rule decay' discussed in [16].

(d) Further increasing $\delta>\delta_{\text {mix }}$, the estimated fidelity decay time eventually becomes smaller than the classical mixing time $t_{\text {mix }}, \tau_{\mathrm{em}}<t_{\text {mix }}$. In this regime, the perturbation is simply too strong so that the fidelity effectively decays within the shortest observable time-scale $\left(t_{\text {mix }}\right)$.

However, if we consider a non-random, e.g. coherent initial state then the quantum correlation function relaxes on a slightly longer, namely Eherenfest time-scale $t_{\mathrm{E}}$ so the regime (c) should terminate already at a little smaller upper border $\delta=\delta_{\mathrm{E}}$ which is naturally determined by the condition $\tau_{\mathrm{em}}=t_{\mathrm{E}}$

$$
\delta_{\mathrm{E}} \approx \hbar \frac{\lambda^{1 / 2}}{\left[\sigma_{\mathrm{cl}} \ln (1 / \hbar)\right]^{1 / 2}} \sim \frac{\delta_{\text {mix }}}{[\ln (1 / \hbar)]^{1 / 2}} .
$$

For coherent initial states one thus obtains an extra but very narrow regime $\delta_{\mathrm{E}}<\delta<$ $\delta_{\text {mix }}$ (describing the time-range $t_{\text {mix }}<t<t_{\mathrm{E}}$ ) where the fidelity decay can be computed in terms of classical Lyapunov exponents [14, 15].

2.3.2. Non-mixing and non-ergodic regime. In this regime things are simpler, as we do not have to worry about the average plateau in the correlation function due to a finite $\mathcal{N}$ because we already have a higher average time correlation $\bar{C} \rightarrow \bar{C}_{\mathrm{cl}} \neq 0$ (12) due 
to non-mixing nature of dynamics. Thus we have here only two relevant time-scales, namely $\tau_{\text {ne }}$ giving initial quadratic decay (13), and the finite size fluctuation time-scale $t^{*}$ (34) which depends on the properties of the initial state (power law (23) for a random initial state, versus gaussian (27) for a coherent initial state). We conjecture, based on a rigorous result in spin $1 / 2$ chains [10], that the fidelity decay in the thermodynamic limit $d \rightarrow \infty$ will generically approach a gaussian (17), which is consistent with increasing power $d / 2$ of power-law fidelity decay (23) for finite dimension $d$.

We can summarize our findings by the following question: Can we have a regime with $\tau_{\mathrm{ne}}<\tau_{\mathrm{em}}$, so that the fidelity will decay faster for a "regular" (non-ergodic) than for a "chaotic" (ergodic and mixing) dynamics? Indeed, if

$$
\delta< \begin{cases}\delta_{\mathrm{r}}:=\hbar \bar{C}_{\mathrm{cl}}^{1 / 2} / \sigma_{\mathrm{cl}} \propto \delta_{\text {mix }} \propto \hbar & \text { random initial state } \\ \delta_{\mathrm{c}}:=\hbar^{3 / 2}\left(\boldsymbol{a}^{\prime} \cdot \Lambda^{-1} \boldsymbol{a}^{\prime}\right)^{1 / 2} /\left(\sqrt{2} \sigma_{\mathrm{cl}}\right) \propto \hbar^{3 / 2} & \text { coherent initial state }\end{cases}
$$

where $\sigma_{\mathrm{cl}}$ and $\bar{C}_{\mathrm{cl}}$ are computed for a mixing and non-ergodic classical dynamics, respectively, then the decay in a non-ergodic case will be faster than in a mixing case. We can see that the condition (44) can be generally satisfied for a random initial state, while for a coherent initial state it can be satisfied above the (second) perturbative border (see fig. 2),$\delta_{\mathrm{c}}>\delta>\delta_{\mathrm{s}}$, only in more than one dimension $d>1$. We note that our result is not contradicting any of the existing findings on quantum-classical

correspondence. For example, a growth of quantum dynamical entropies [18 persists only up to logarithmically short Ehrenfest time $t_{\mathrm{E}}$, which is the upper bound for the validity of another semiclassical approach to the fidelity decay [14, 15] and within which one would always find $F_{\text {ne }}^{\text {coh }}(t)>F_{\text {em }}(t)$ above the perturbative border $\delta>\delta_{\mathrm{p}}$, whereas our theory reveals new nontrivial quantum phenomena with a semiclassical prediction (but not correspondence!) much beyond that time. If we let $\hbar \rightarrow 0$ first, and then $\delta \rightarrow 0$, i.e. we keep $\delta \gg \delta_{\mathrm{r}, \mathrm{c}}(\hbar)$, then we recover a result supported by a classical intuition, namely that the regular (non-ergodic) dynamics is more stable than the chaotic (ergodic and mixing) dynamics. On the other hand, if we let $\delta \rightarrow 0$ first, and only after that $\hbar \rightarrow 0$, i.e. satisfying (44), we find somewhat counterintuitive results saying that chaotic (mixing) dynamics is more stable than the regular one. We can conclude the section by saying that we have three non-commuting limits, namely the semiclassical limit $\hbar \rightarrow 0$ the perturbation strength limit $\delta \rightarrow 0$, and the thermodynamic limit $d \rightarrow \infty$, such that no pair of these limits commutes.

\section{Numerical experiments}

\subsection{Kicked top}

Here we wish to verify and demonstrate the results of the previous section by numerical experiments. For this purpose we choose the Haake's quantized kicked top [19] since this model served as a model example for many related studies [8, 16, 18, 20, 21, 22, 23]. 
The unitary propagator reads

$$
U=U(\alpha, \gamma)=\exp \left(-i \gamma J_{\mathrm{y}}\right) \exp \left(-i \alpha J_{\mathrm{z}}^{2} / 2 J\right),
$$

where $J_{k}(k=\mathrm{x}, \mathrm{y}, \mathrm{z})$ are quantum angular momentum operators, $\left[J_{k}, J_{l}\right]=i \epsilon_{k l r} J_{r}$. (Half)integer $J$ determines the size of the Hilbert space $2 J+1$ and the value of effective Planck's constant $\hbar=1 / J$. The perturbation is defined by perturbing the parameter $\alpha, U_{\delta}=U(\alpha+\delta, \gamma)$, so that the generator $A$ is

$$
A=\frac{1}{2}\left(\frac{J_{\mathrm{z}}}{J}\right)^{2} \text {, }
$$

Physically, the system (45) represents a twist around z-axis followed by a rotation for an angle $\gamma$ around y-axis. The classical limit is obtained by letting $J=1 / \hbar \rightarrow \infty$ and writing the classical angular momentum in terms of a unit vector on a sphere $\boldsymbol{r}=(x, y, z)=\boldsymbol{J} / J$. The Heisenberg equation for the SU(2) operators $\boldsymbol{J}, \boldsymbol{J}^{\prime}=U^{\dagger} \boldsymbol{J} U$, reduces to the classical area preserving map of a sphere

$$
\begin{aligned}
& x^{\prime}=\cos \gamma(x \cos \alpha z-y \sin \alpha z)+z \sin \gamma \\
& y^{\prime}=y \cos \alpha z+x \sin \alpha z \\
& z^{\prime}=z \cos \gamma+\sin \gamma(y \sin \alpha z-x \cos \alpha z) .
\end{aligned}
$$

Note that in the classical limit the perturbation generator is

$$
a(\boldsymbol{r})=\frac{z^{2}}{2} .
$$

For $\alpha=0$ the system is integrable, while with increasing $\alpha$ there is a transition to chaotic motion. The second parameter $\gamma$ is usually set to $\pi / 2$, however in our numerical simulation we will use two different values exhibiting qualitatively different correlation decay (for large $\alpha$ ): the 'standard' case $\gamma=\pi / 2$ where $C_{\mathrm{cl}}(t)$ decays in oscillatory way and the case $\gamma=\pi / 6$ where $C_{\mathrm{cl}}(t)$ decays monotonically (see fig. 3).

In the case of $\gamma=\pi / 2$ we have two discrete symmetries. The evolution $U$ commutes with $R_{\mathrm{x}}$ and $R_{\mathrm{y}}$, the rotations for $\pi$ around $\mathrm{x}$ and y axes, respectively. The Hilbert space is therefore reducible into three invariant subspaces (using notation of Peres's book [2] with the basis $|m\rangle$ of eigenstates of $J_{z}$ and assuming $J$ to be an even integer): EE of dimension $J / 2+1$ with the basis states $|0\rangle$ and $\{|2 m\rangle+|-2 m\rangle\} / \sqrt{2}$; OO of dimension $J / 2$ with the basis $\{|2 m-1\rangle-|-(2 m-1)\rangle\} / \sqrt{2}$; OE of dimension $J$ with the basis $\{|2 m\rangle-|-2 m\rangle\} / \sqrt{2}$ and $\{|2 m-1\rangle+|-(2 m-1)\rangle\} / \sqrt{2}$ with $m$ in all three cases running through $m=1, \ldots, J / 2$. For $\gamma \neq \pi / 2$ the spaces OO and EE coalesce as $R_{\mathrm{y}}$ is the only discrete symmetry left. In numerical experiments we always choose OE subspace so that the dimension of the Hilbert space is $\mathcal{N}=J$.

We will compute the fidelity using three different statistical operators $\rho$ : (1) $\rho=\mathbb{1} / \mathcal{N}$ corresponding to full Hilbert space average, (2) pure random initial state $\rho=|\psi\rangle\langle\psi|$ (components $c_{m}=\langle m \mid \psi\rangle$ being independent gaussian pseudo-random numbers) giving the same results as (1) however with a higher finite size fluctuation plateau $F_{\text {rmt }}$ (as discussed in subsect. 2.3), and (3) pure minimal wavepacket initial 
state $\rho=|\vartheta, \varphi\rangle\langle\vartheta, \varphi|$, namely $\mathrm{SU}(2)$ coherent state $|\vartheta, \varphi\rangle$ centered at the point $\boldsymbol{n}=(\sin \vartheta \cos \varphi, \sin \vartheta \sin \varphi, \cos \vartheta)$ on a unit sphere

$$
|\vartheta, \varphi\rangle=\sum_{m=-J}^{J}\left(\begin{array}{c}
2 J \\
J+m
\end{array}\right)^{1 / 2} \cos (\vartheta / 2)^{J+m} \sin (\vartheta / 2)^{J-m} e^{-i m \varphi}|m\rangle .
$$

3.1.1. Mixing regime. In this regime we will demonstrate two main different decays of fidelity as discussed in subsect. 2.3, namely the exponential decay $F_{\mathrm{em}}(t)$ (11) for $\delta_{\mathrm{s}}<\delta<\delta_{\text {mix }}$ and a finite size (perturbative) gaussian decay $F_{\mathrm{p}}(t)$ (40) for a small perturbation $\delta<\delta_{\mathrm{p}}$. We choose large $\alpha=30$ to ensure fast mixing. This value seems to be excessively large, but for smaller $\alpha$ we still have "sticky" structures in classical phase space which in turn cause slow algebraic tails in the classical correlation function. We must remember that the derivation of an exponential decay required well defined mixing time-scale $t_{\text {mix }}$ which is not the case if we have a slow power-law decay of correlations.

Exponential decay time $\tau_{\mathrm{em}}$ (11) is determined by an integral/sum of time correlation function (9), which can be calculated in the semiclassical regime $\left(\sigma_{\mathrm{cl}}\right)$ by means of the correlation function of the classical map

$$
C_{\mathrm{cl}}(t)=\frac{1}{4}\left\langle\tilde{z}^{2}(0) \tilde{z}^{2}(t)\right\rangle .
$$

$\tilde{z}^{2}=z^{2}-\left\langle z^{2}\right\rangle$ is a "traceless" perturbation where averaging over the sphere gives $\left\langle z^{2}\right\rangle=1 / 3$. The classical correlation functions calculated by using the classical map (47) are shown in fig. 3. For $\gamma=\pi / 2$ the correlation function is oscillating with an exponential envelope hence the transport coefficient $\sigma_{\mathrm{cl}}=0.00385$ is quite small. For $\gamma=\pi / 6$ the correlation decay is monotonic and exponential with $\sigma_{\mathrm{cl}}=0.0515$. The decay of quantum fidelity (11) can now be obtained by using the classical limit $\sigma \rightarrow \sigma_{\mathrm{cl}}$ :

$$
F_{\mathrm{em}}(t)=\exp \left(-\delta^{2} J^{2} \sigma_{\mathrm{cl}} t\right),
$$

This formula has been compared with the exact numerical calculation of fidelity (2) where averaging over the whole Hilbert space has been employed, i.e. $\rho=\mathbb{1} / \mathcal{N}$, and, as expected due to ergodicity, there was no difference for sufficiently large $J$ when we have chosen a fixed coherent initial state. As the finite size fidelity fluctuation level $F_{\text {rms-iii }}(33)$ decreases with increasing Hilbert space dimension, we chose large $J=4000$ in order to be able to check exponential decay (51) over as many orders of magnitude as possible. The results are shown in fig. 1 . The smallest and the largest $\delta$ shown roughly correspond to borders $\delta_{\mathrm{s}}$ and $\delta_{\mathrm{mix}}$, respectively. As we can see, the agreement with an exponential decay is excellent, at least over four decades in probability $|F(t)|^{2}$. Note that for $\gamma=\pi / 2$ and the largest $\delta=3 \cdot 10^{-3}$ the time-scale of the decay of fidelity is comparable to the classical time-scale $t_{\text {mix }}$ so the factorization assumption (10) is strictly not applicable any more. However, due to oscillatory correlation decay, overall agreement with the theory (51) is still rather good but the oscillations in the correlation decay reflect in the oscillations of the fidelity decay (around the theoretical exponential curve). Of course, one does not need such a large $J$ in order to have an exponential 


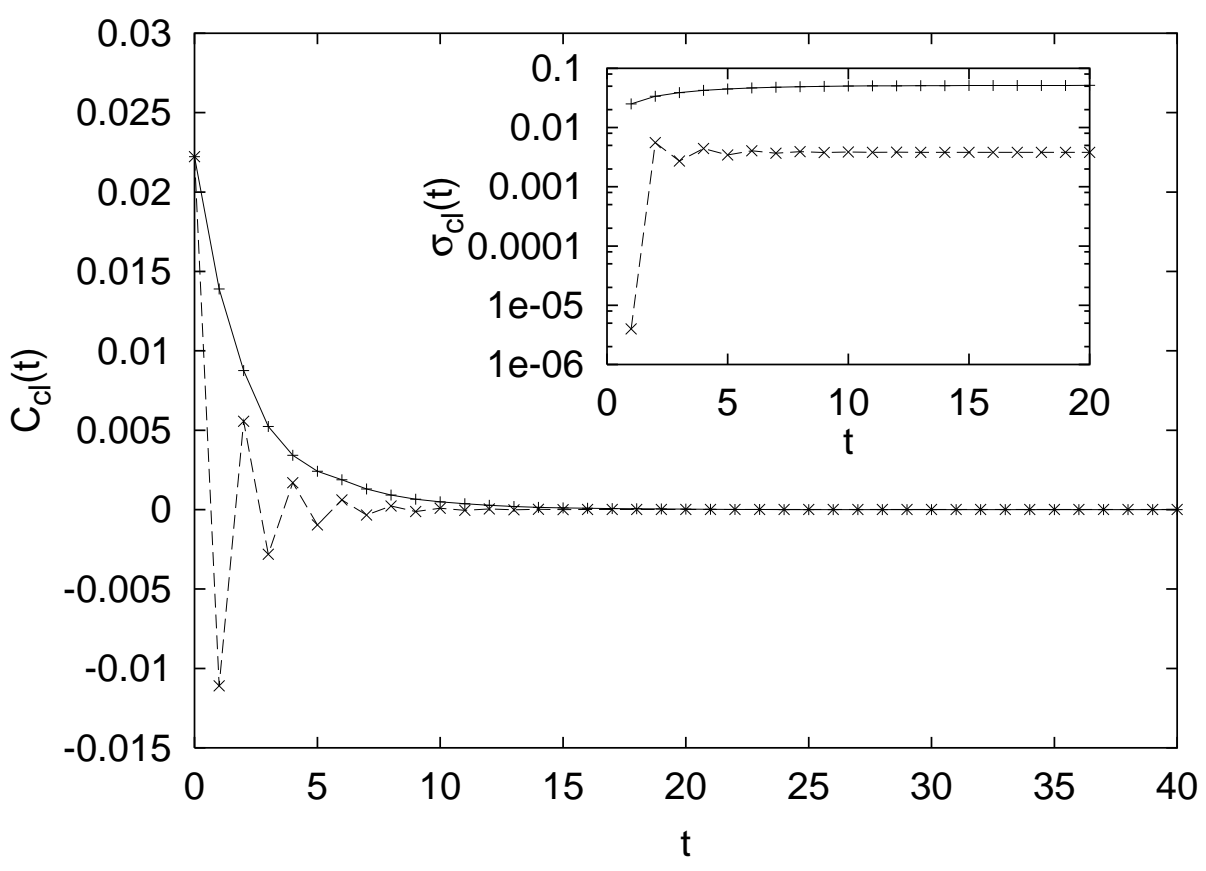

Figure 3. Classical correlation function $C_{\mathrm{cl}}(t)$ (50) for $\alpha=30$, and $\gamma=\pi / 6$ (top, full curve) and $\gamma=\pi / 2$ (bottom, broken curve). Finite time integrated correlation function is shown in the inset, converging to $\sigma_{\mathrm{cl}}=0.00385$ and 0.0515 , for $\gamma=\pi / 2$ and $\pi / 6$, respectively. Averaging over $10^{5}$ random initial conditions on a sphere is performed.

decay, but for smaller $J$ the fluctuation level $F_{\text {rms }}$ will be higher so the exponential decay (51) will persist for correspondingly smaller time, namely up to $t_{*}(J)$ (34).

Then we focus on the so-called perturbative regime $\delta<\delta_{\mathrm{p}}$ where the fidelity decay will be dictated by a finite size correlation average (37), so according to eq. (40)

$$
F_{\mathrm{p}}(t)=\exp \left(-2 \delta^{2} J \sigma_{\mathrm{cl}} t^{2}\right) .
$$

We numerically computed $\bar{C}$ (12) for $J=1500, \alpha=30$ in order to show that it is given by the theoretical value (37). Quantum correlation function has been computed $C\left(t^{\prime}, t^{\prime \prime}\right)=$ $\left\langle\tilde{A}_{t^{\prime}} \tilde{A}_{t^{\prime \prime}}\right\rangle$ by means of a traceless perturbation $\tilde{A}=\frac{1}{2}\left(J_{z} / J\right)^{2}-\frac{1}{12}\left[(2 J+1)(J+1) / J^{2}\right] \mathbb{1}$. In fig. 5 we show a finite time correlation sum $\sigma(t)=\frac{1}{2 t} \sum_{t^{\prime}, t^{\prime \prime}=0}^{t-1} C\left(t^{\prime}, t^{\prime \prime}\right)$ which exhibits a crossover, at the Heisenberg time $t_{\mathrm{p}}=J / 2$, from the plateau given by $\sigma_{\mathrm{cl}}$ to a linear increase $\bar{C} t$ due to finite size correlation average (37) $\bar{C}=4 \sigma_{\mathrm{cl}} / J$. The excellent agreement between prediction (52) and full numerical calculation of fidelity is shown in fig. 6. In view of our findings this so-called [16] perturbative regime can be understood as a simple consequence of a finite Hilbert space dimension. For times larger than the Heisenberg time $t_{\mathrm{p}}$ every quantum system behaves effectively as an integrable one, e.g. with a finite time average correlation plateau.

3.1.2. Non-mixing regime. In a kicked top this regime is realized for sufficiently small value of $\alpha$. If the classical phase space has a mixed (KAM) structure, non- 

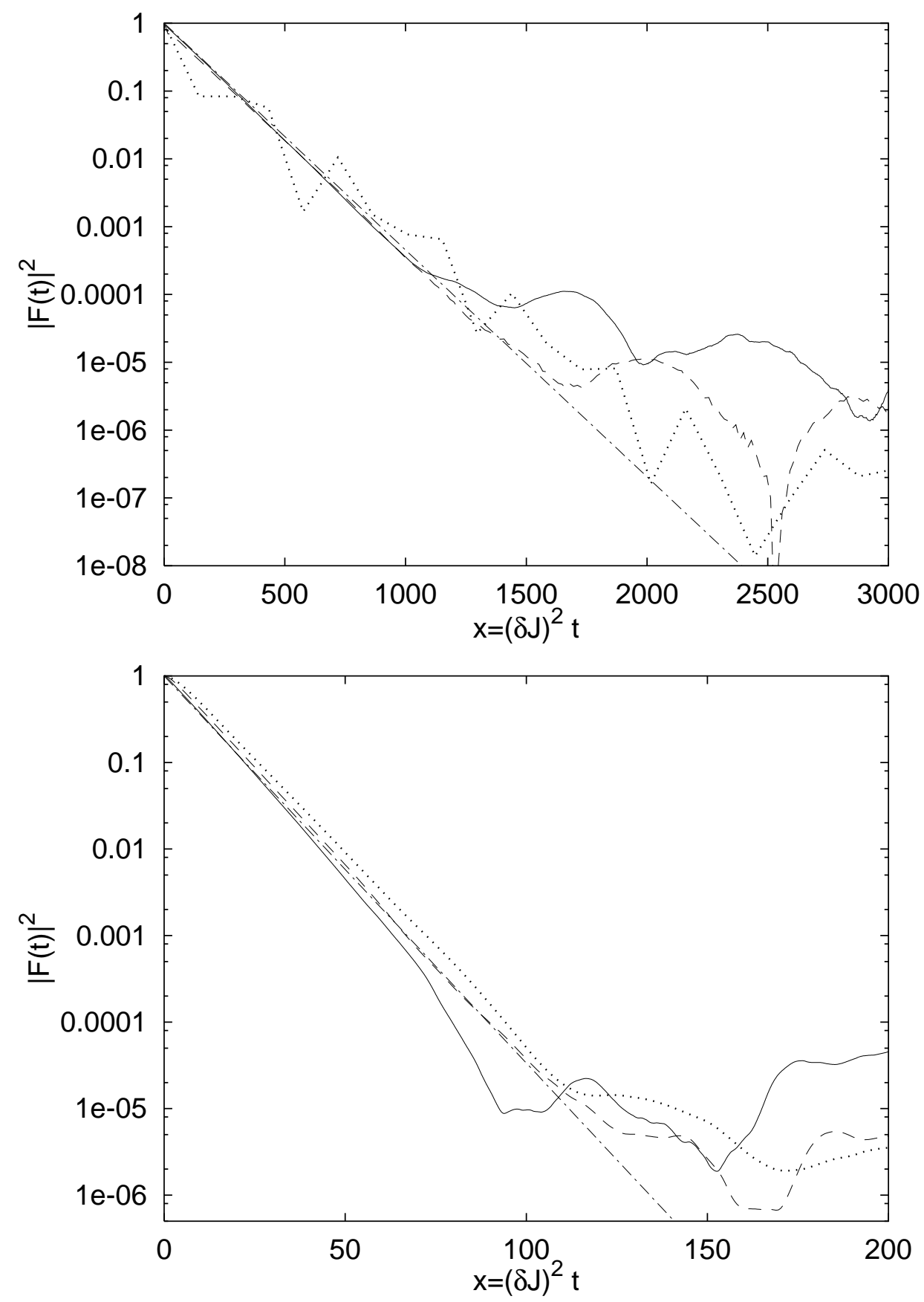

Figure 4. Quantum fidelity for a kicked top with parameters $\alpha=30, J=4000$ and full trace average. Top figure is for $\gamma=\pi / 2$ and for $\delta=5 \cdot 10^{-4}, 1 \cdot 10^{-3}, 3 \cdot 10^{-3}$ (solid, dashed, dotted curves, respectively) Bottom figure is for $\gamma=\pi / 6$ and $\delta=$ $1 \cdot 10^{-4}, 2 \cdot 10^{-4}, 3 \cdot 10^{-4}$, (solid, dashed, dotted curves, respectively). Chain line in both cases gives the theory (51) with classically computed $\sigma_{\mathrm{cl}}$. Note that the largest $\delta=3 \cdot 10^{-3}$ case in the top figure (dotted curve) corresponds to $\tau \approx 2$, so it is already over the upper border of the regime (c, subsect. 2.3) $\delta>\delta_{\operatorname{mix}}$ but the agreement with the theory (51) is still quite good, appart from oscillations. This is due to the oscillatory nature of time-correlations making the factorization assumption (10) justified (on average) even for much smaller time $t$ as required. 


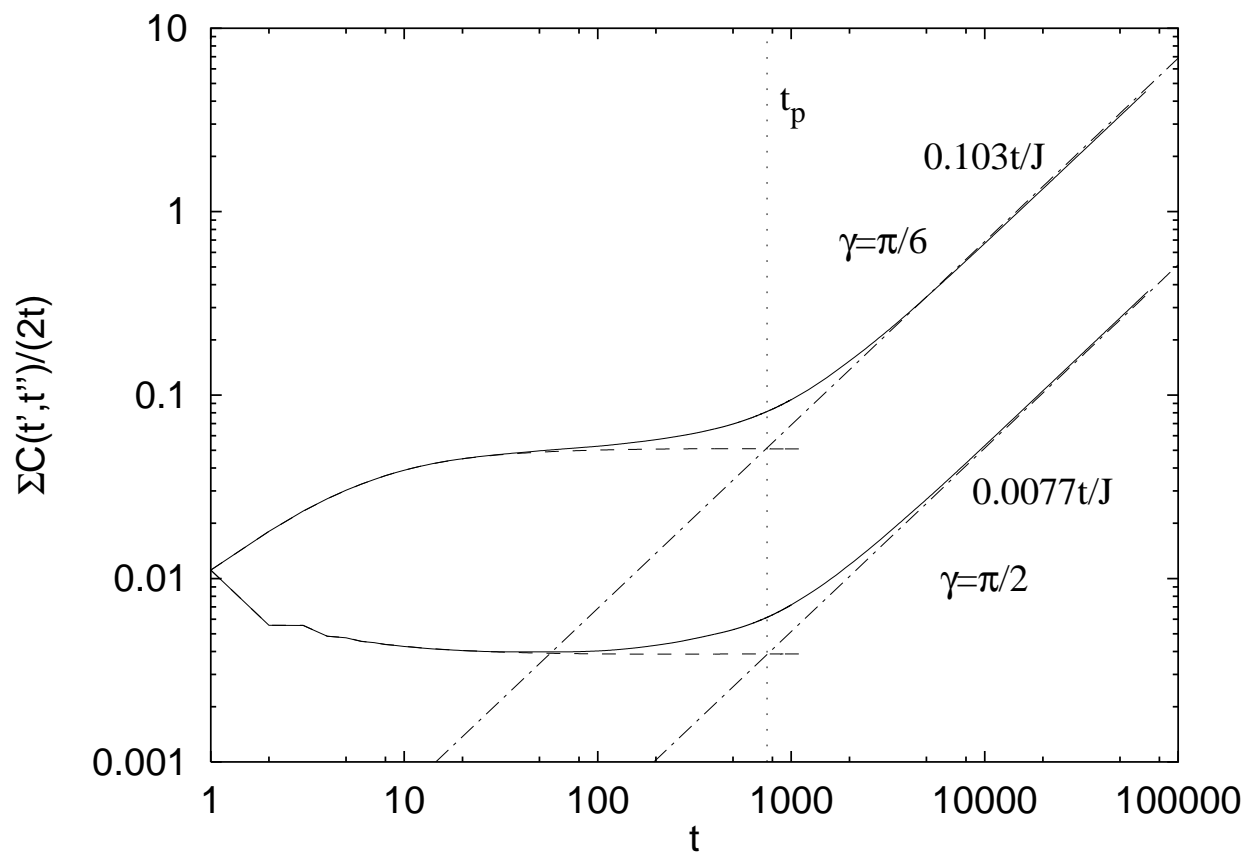

Figure 5. Finite time quantum correlation sum $\sigma(t)=\sum_{t^{\prime}, t^{\prime \prime}=0}^{t-1} C\left(t^{\prime}, t^{\prime \prime}\right) / 2 t$ (solid curves) and the corresponding classical sum $\sigma_{\mathrm{cl}}(t)=\sum_{t^{\prime}, t^{\prime \prime}=0}^{t-1} C_{\mathrm{cl}}\left(t^{\prime}, t^{\prime \prime}\right) / 2 t$ (dashed curves saturating at $\sigma_{\mathrm{cl}}$ and ending at $t \sim 1000$ ) for $\alpha=30, J=1500$. Upper curves are for $\gamma=\pi / 6$ while lower curves are for $\gamma=\pi / 2$. Chain lines are best fitting asymptotic linear functions corresponding to $\bar{C} t, 0.0077 t / J$ for $\gamma=\pi / 2$ and $0.103 t / J$ for $\gamma=\pi / 6$.

mixing regime of fidelity decay may be obtained by choosing a localized initial state (e.g. coherent state) located in a regular part of the phase space. However, such a situation may easily lead to the opposite conclusion (as compared to generic situation) for insufficiently large dimension $\mathcal{N}$. As discussed in subsect.2.3, the fidelity fluctuation plateau is determined by the number of constituent propagator eigenstates $\left|\phi_{n}\right\rangle$ which are effectively needed to expand the initial state. For a coherent state sitting inside a (not too large) regular (KAM) island this number can be fairly small for numerically realizable Hilbert space dimensions, thus prohibiting any significant fidelity decay as observed in [5]. Nevertheless, we would still see the initial quadratic decay in the linear response regime but we would not be able to verify higher orders in the long-time expansion of fidelity. Therefore, in order to make a situation numerically as clean as possible, we choose a small value of parameter $\alpha=0.1$, such that the classical dynamics is almost integrable and that the majority of phase space corresponds to regular motion so that the number of constituent eigenstates for coherent states is as large as possible (on average).

Here we focus only on the case $\gamma=\pi / 2$. For small $\alpha$, the quantum and classical evolution is a (slightly perturbed) rotation around $\mathrm{y}$ axis and the time averaged 


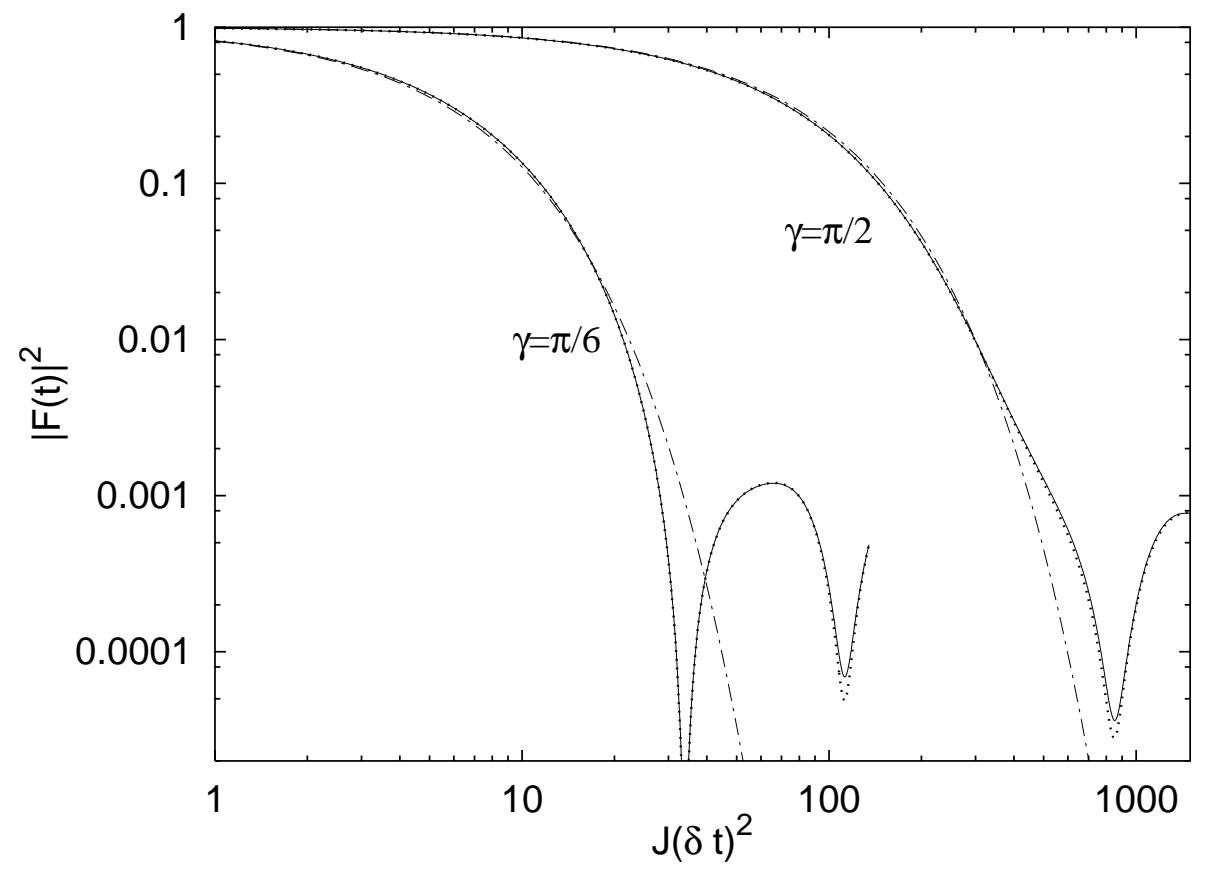

Figure 6. Quantum fidelity in the perturbative regime $\delta<\delta_{\mathrm{p}}$ for $\alpha=30, J=1500$, and $\gamma=\pi / 2$ and $\pi / 6$, calculated as a full trace Hilbert space average as a function of the scaled variable $J(\delta t)^{2}$. For $\gamma=\pi / 2$ data for $\delta=1 \cdot 10^{-6}$ (solid curve) and $5 \cdot 10^{-6}$ (dotted curve) are shown. For $\gamma=\pi / 6, \delta=3 \cdot 10^{-7}$ (solid) and $1 \cdot 10^{-6}$ (dotted) are shown. Note that for both $\gamma$ the curves for both $\delta$ practically overlap. The chain curves are theoretical predictions (52) with classically computed $\sigma_{\mathrm{cl}}$.

perturbation can be computed analytically (in the leading order in $\alpha$ )

$$
\bar{A}=\frac{1}{4 J^{2}}\left(J_{\mathrm{z}}^{2}+J_{\mathrm{x}}^{2}\right)=\frac{1}{4}\left(1-\left(J_{\mathrm{y}} / J\right)^{2}\right), \quad \bar{a}=\frac{1}{4}\left(1-y^{2}\right) .
$$

We will now use (approximate) analytical results for $\alpha \rightarrow 0$ to compare with numerics for $\alpha=0.1$. It should be noted that our leading order analytical approximations could easily be systematically improved using a classical perturbation theory (treating $\alpha$ as a perturbing parameter). However, since the agreement, as shown below, is almost perfect in all cases, we see no need for refinement at this level.

First, we consider the full trace average, $\rho=\mathbb{1} / J$, and starting from the expression (15), $F(t)=\langle\exp (i t \bar{A} \delta / \hbar)\rangle$, write the fidelity as a sum over all eigenvalues of $J_{\mathrm{y}}^{2}$, namely $(2 m-1)^{2}$, for $m=1, \ldots, J / 2$ (in OE subspace),

$$
|F(t)|=\left|\frac{2}{J} \sum_{m=1}^{J / 2} \exp \left(i \delta t(2 m-1)^{2} / 4 J\right)\right| .
$$

For large $J$ we can replace the sum with an integral and get

$$
|F(t)|=\sqrt{\frac{\pi}{\delta J t}}\left|\operatorname{erfi}\left(\frac{1}{2} e^{i \pi / 4} \sqrt{\delta J t}\right)\right|
$$

where $\operatorname{erfi}(z)=\frac{2}{i \sqrt{\pi}} \int_{0}^{i z} e^{-t^{2}} d t$ is a complex error function with a limit $\lim _{x \rightarrow \infty}\left|\operatorname{erfi}\left(\frac{1}{2} e^{i \pi / 4} \sqrt{x}\right)\right|=1$ to which it approaches by oscillating around 1 . We therefore have an analytic expression for the fidelity (55) in the case of an uniform average 


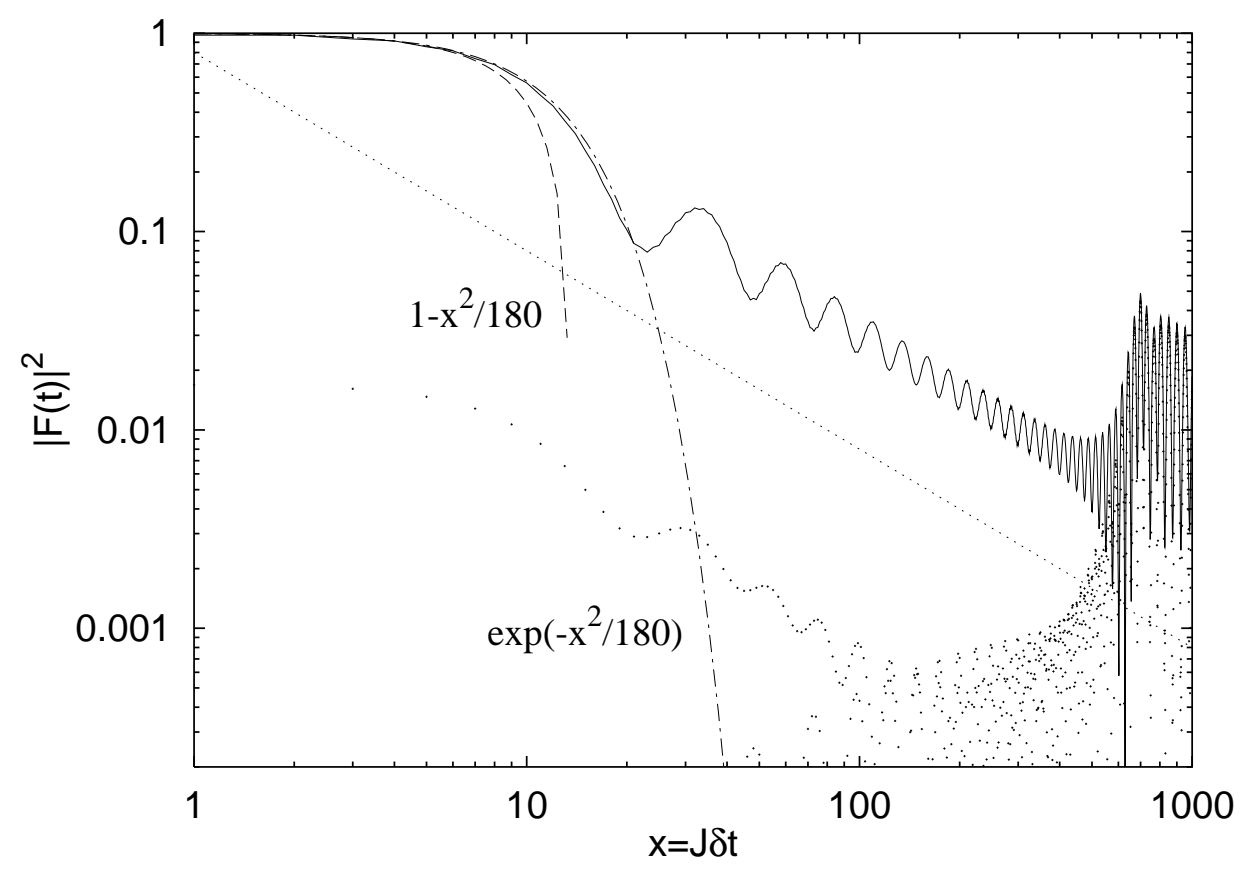

Figure 7. Fidelity in quasi-integrable regime for $\alpha=0.1, \gamma=\pi / 2, \delta=0.01, J=100$, and $\rho=\mathbb{1} / J$, in OE subspace. Solid curve gives the result of a numerical simulation. Isolated dots denote differences between numerical calculation and the analytic formula (55) for $\left.\alpha \rightarrow 0|| F_{\text {num. }}(t)\right|^{2}-\left|F_{\text {anali. }}(t)\right|^{2} \mid$. The dotted line gives a predicted asymptotic decay $\propto t^{-1 / 2}$, and the dashed/chain curves are the predicted fidelity decays at small times, namely the second order expansion $|F(t)|^{2}=1-(J t \delta)^{2} / 180$, and 'improved' by the gaussian (17).

over whole Hilbert space or, equivalently, over one random initial state. Its asymptotic decay is $t^{-1 / 2}$ which agrees with the general semiclassical asymptotics (23) and we expect initial quadratic decay (13) for small times $t<\tau_{\text {ne }}$ Decay rate $\tau_{\text {ne }}$ is determined by the time averaged correlation $\bar{C}$ (12) which can be calculated explicitly in the limit $\alpha \rightarrow 0$ where the classical correlation function $C_{\mathrm{cl}}(t)$ alternates for even/odd times as $C_{\mathrm{cl}}(2 t)=\left\langle\tilde{z}^{2}(0) \tilde{z}^{2}(2 t)\right\rangle / 4=-1 / 90, C_{\mathrm{cl}}(2 t+1)=\left\langle\tilde{z}^{2}(0) \tilde{z}^{2}(2 t+1)\right\rangle / 4=1 / 45$, giving

$$
\left.\bar{C}_{\mathrm{cl}}\right|_{\alpha=0}=\frac{1}{2}\left(-\frac{1}{90}+\frac{1}{45}\right)=\frac{1}{180},
$$

and the fidelity is expected to decay as (13) with $\tau_{\text {ne }}=\sqrt{180} /(J \delta)$, for short times, $t<\tau_{\text {ne. }}$. The short-time formula (13) and the full analytic expression (55) are compared with the numerical simulation in fig. 7 . The agreement is very good and, surprisingly enough, the gaussian approximation (117) for small times is observed to be valid considerably beyond the second order expansion (13). Quite interesting is the regime where the decay time $\tau_{\text {ne }}=\sqrt{180} /(J \delta)$ for a "regular" dynamics will be smaller than a decay time $\tau_{\mathrm{em}}=1 /\left(\delta^{2} J^{2} \sigma_{\mathrm{cl}}\right)$ (51) for a "chaotic" dynamics. This will happen for $\delta<\delta_{\mathrm{r}}=1 /\left(J \sigma_{\mathrm{cl}} \sqrt{180}\right)$ (44). This border has the same scaling with $J$ as $\delta_{\text {mix }}$ (42).

Second, considering SU(2) coherent initial state (49), we could proceed along the same line, namely by an analytic calculation. But rather than that, we will illustrate 


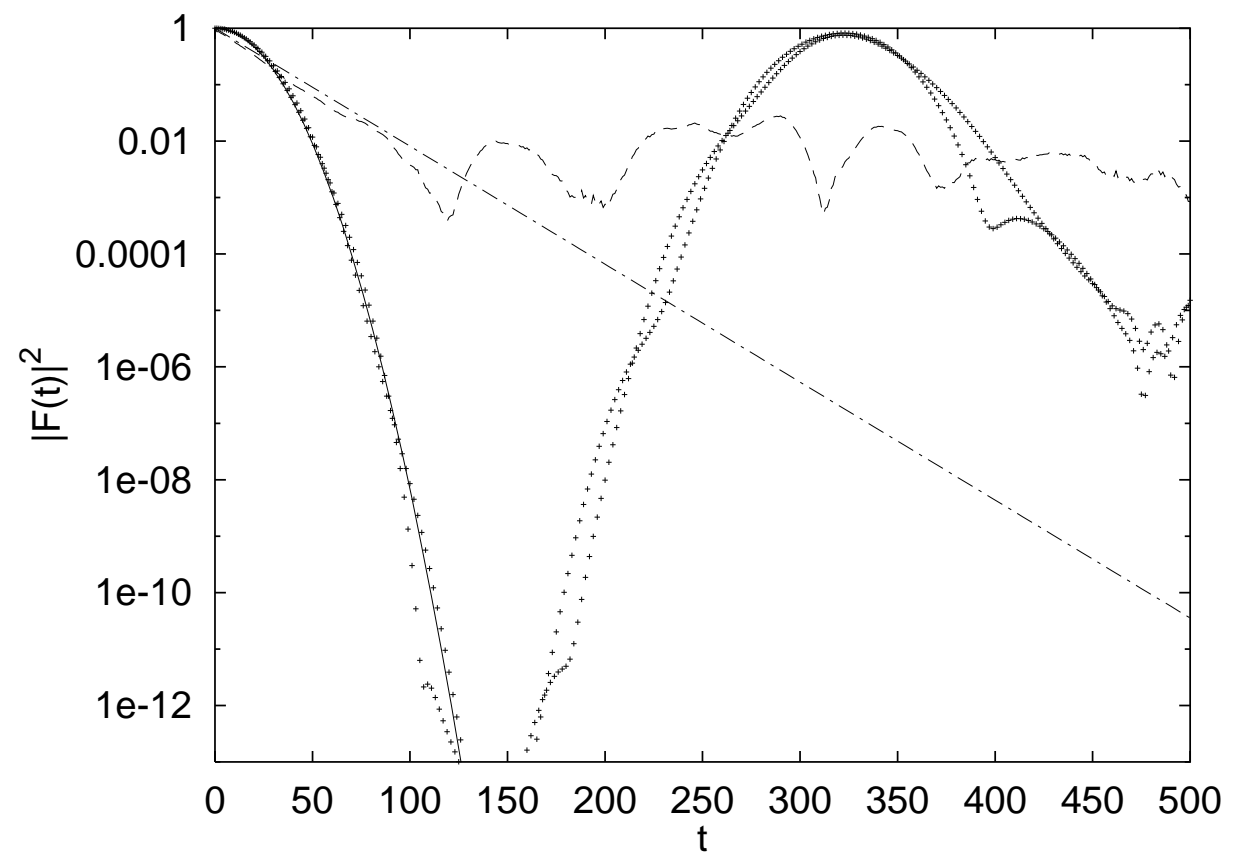

Figure 8. Fidelity for $\gamma=\pi / 2, \delta=0.025$ and $J=100$ on OE subspace. The dashed curve is a simulation for $\alpha=30$ (mixing regime, full trace average). The pluses are for a pure coherent initial state (see text for details) at $\alpha=0.1$ (non-mixing regime). The chain and solid curves are, respectively, the theoretical exponential (51) and gaussian (59) decays.

the usefulness of a semiclassical formula for $F_{\text {ne }}^{\text {coh }}(t)$ (27). This is a more general approach, as the explicit analytical calculation is usually not possible. Let us by $\tilde{\vartheta}, \tilde{\varphi}$ denote the spherical angular coordinates measured with respect to the y-axis. Then $(I=\cos \tilde{\vartheta}=y, \tilde{\varphi})$ represent canonical action-angle coordinates for the integrable case $\alpha \rightarrow 0$. Furthermore, the coherent state (49) acquires a semiclassical gaussian form (24) in the EBK basis $|n\rangle, J_{\mathrm{y}}|n\rangle=n|n\rangle$, namely

$$
|\langle n \mid \tilde{\vartheta}, \tilde{\varphi}\rangle|^{2} \propto \exp \left(-\frac{(n \hbar-\cos \tilde{\vartheta})^{2}}{\hbar \sin ^{2} \tilde{\vartheta}}\right), \quad \hbar=\frac{1}{J} .
$$

The squeezing parameter $\Lambda$ reads

$$
\Lambda=1 / \sin ^{2} \tilde{\vartheta}=1 /\left(1-y^{2}\right) .
$$

In order to apply the general formula (27) we need to express the classical time average (53) in terms of a canonical action, $\bar{a}(I)=\left(1-I^{2}\right) / 4$, and evaluate the derivative, $|\partial \bar{a}(I) / \partial I|^{2}=\frac{1}{4} I^{2}$, giving $\left|F_{\mathrm{ne}}^{\mathrm{coh}}(t)\right|=\exp \left(-\delta^{2} J t^{2} I^{2}\left(1-I^{2}\right) / 16\right)$. Rewriting this expression in terms of original spherical angles $\vartheta$ and $\varphi$ measured with respect to zaxis, we obtain

$$
F_{\mathrm{ne}}^{\mathrm{coh}}(t)=\exp \left(-\frac{\delta^{2} J t^{2}}{64}\left\{\sin ^{2} 2 \vartheta \sin ^{2} \varphi+\sin ^{4} \vartheta \sin ^{2} 2 \varphi\right\}\right) .
$$

Here the same interesting question, namely when do we have $\tau_{\text {ne-coh }}<\tau_{\text {em }}$, results in a condition (44) $\delta<1 /\left(4 \sigma_{\mathrm{cl}} \sqrt{2 J^{3}}\right)=\delta_{\mathrm{s}} / \sqrt{32 \sigma_{\mathrm{cl}} \ln J}$ (we did not write the factor 
involving trigonometric functions (59) which is of order 1). This condition generally can not be meet if we also require $\delta>\delta_{\mathrm{s}}$ (in order to see full exponential decay in the mixing regime), except if $32 \sigma_{\mathrm{cl}} \ln J<1$. This is for instance satisfied for $J=100$ and $\gamma=\pi / 2$, for which $\sigma_{\mathrm{cl}}=0.00385$. We checked this by a numerical simulation and the result for a coherent state centered at $(\vartheta, \varphi)=\pi(1 / \sqrt{3}, 1 / \sqrt{2})$, for which $\sin ^{2} 2 \vartheta \sin ^{2} \varphi+\sin ^{4} \vartheta \sin ^{2} 2 \varphi=0.96$, is shown in fig. \&. We can see that for $t>50$ the fidelity in a non-mixing regime $\left(\tau_{\text {ne-coh }}=23\right)$ is lower than the fidelity in a mixing regime $\left(\tau_{\mathrm{em}}=42\right)$. For larger times, $t>t^{*}$, non-mixing decay $F_{\mathrm{ne}}^{\text {coh }}$ displays revivals of fidelity.

Finally, we want to visualize the phenomenon of faster decay of fidelity in a regular, non-mixing regime $\alpha=0.1$ in comparison with the chaotic, mixing regime $\alpha=30$ by using the phase space representation of wave functions. A convenient and popular choice is a Husimi function $H(\varphi, \cos \vartheta)$ of a state $|\psi\rangle$ defined as

$$
H(\varphi, \cos \vartheta)=|\langle\vartheta, \varphi \mid \psi\rangle|^{2} .
$$

We have chosen a pure random initial state $|\psi\rangle$ and propagated it, in the first case with the propagator $U$ for $\alpha=0.1$, and in the second case for $\alpha=30$, as well as with the perturbed propagators $U_{\delta}$ in both cases. The state and all the other parameters (e.g. perturbation strength $\delta$ ) were identical in both cases. Then we compared the differences between the Husimi functions $\Delta H(\varphi, \cos \vartheta)=H(\varphi, \cos \vartheta)-H_{\delta}(\varphi, \cos \vartheta)$ of the perturbed and the unperturbed time evolution. The results for $\alpha=0.1$ are shown in fig. 9 and for $\alpha=30$ in fig. 10. For chosen $\delta=10^{-4}$ the fidelity decay for the regular dynamics is much faster than for the chaotic one and this effect can also be observed in Husimi functions by comparing the right columns of both figures. But one should stress that by considering the difference of Husimi functions $\Delta H=H-H_{\delta}$, we loose information about the relative phases of the perturbed and unperturbed wave functions in the coherent-state basis which are more important for the fidelity decay in the regular case $(\alpha=0.1)$. However, results shown in figs. 9,10 suggest that not only the quantum phases but also the amplitudes (in some classically motivated, e.g. coherent state basis) exhibit larger susceptibility to system perturbations for the regular (non-mixing) as compared to the chaotic (mixing) dynamics.

\subsection{Pair of coupled kicked tops}

As we have already remarked at the end of sect. 2.3, for a one dimensional $d=1$ systems, the 'surprising' behavior $\tau_{\text {ne-coh }}<\tau_{\text {em }}$ is for coherent initial states possible only around the border (41) $\delta_{\mathrm{s}}$ (unless $\sigma_{\mathrm{cl}}$ is very small like in the example of fig. 8) where the exponential decay in the mixing regime goes over to a gaussian due to finite size $\mathcal{N}$. However, for more than one degree of freedom, $d>1$, such behavior is generally possible well above the finite size - perturbative border $\delta_{\mathrm{s}}$. In order to illustrate this phenomenon we will now briefly consider a numerical example of a pair of coupled kicked tops [24] where $d=2$. 
Stability of quantum motion and correlation decay

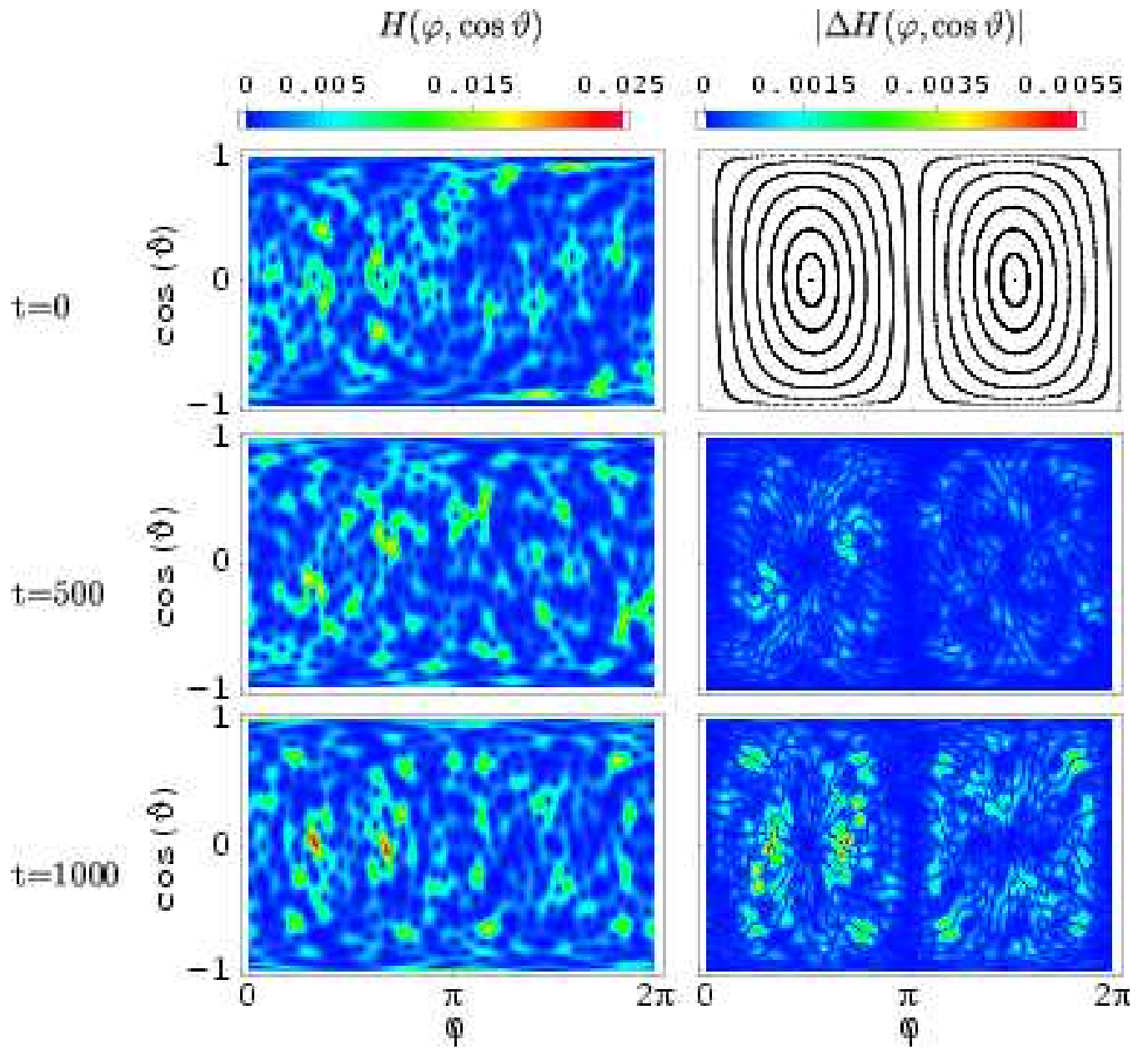

Figure 9. Husimi function $H(\varphi, \cos \vartheta)$ for a regular evolution $\alpha=0.1, \gamma=\pi / 2$ and three different times $t=0,500$ and 1000 (left column). Gaussian random initial state is used and $J=200, \delta=10^{-4}$. In the right column we show pictures of absolute difference between Husimi functions of the unperturbed and perturbed time evolution, $\left|H(\varphi, \cos \vartheta)-H_{\delta}(\varphi, \cos \vartheta)\right|$. Fidelity at the times shown is $|F(500)|=0.73$ and $|F(1000)|=0.25$. Top picture in the right column gives the classical phase space portrait.

We consider coupled kicked tops with a unitary propagator (a simplified version of that of ref. 24])

$$
U(\epsilon)=e^{-i \frac{\pi}{2} J_{1 \mathrm{y}}} e^{-i \frac{\pi}{2} J_{2 \mathrm{y}}} e^{-i \epsilon J_{1 \mathrm{z}} J_{2 \mathrm{z}} / J} .
$$

where $\boldsymbol{J}_{\mathbf{1}}$ and $\boldsymbol{J}_{\mathbf{2}}$ are two independent quantum angular momentum vectors. Perturbed propagator is obtained by perturbing the parameter $\epsilon$, so that $U_{\delta}=U(\epsilon+\delta)$. The perturbation generator is therefore

$$
A=\frac{1}{J^{2}} J_{1 \mathrm{z}} J_{2 \mathrm{z}}
$$

with $\hbar=1 / J$. A pair of coupled kicked tops possesses the same pair of discrete symmetries as the single top for $\gamma=\pi / 2$ [2], namely $R_{\mathrm{x}}$ and $R_{\mathrm{y}}$, and in addition 
Stability of quantum motion and correlation decay

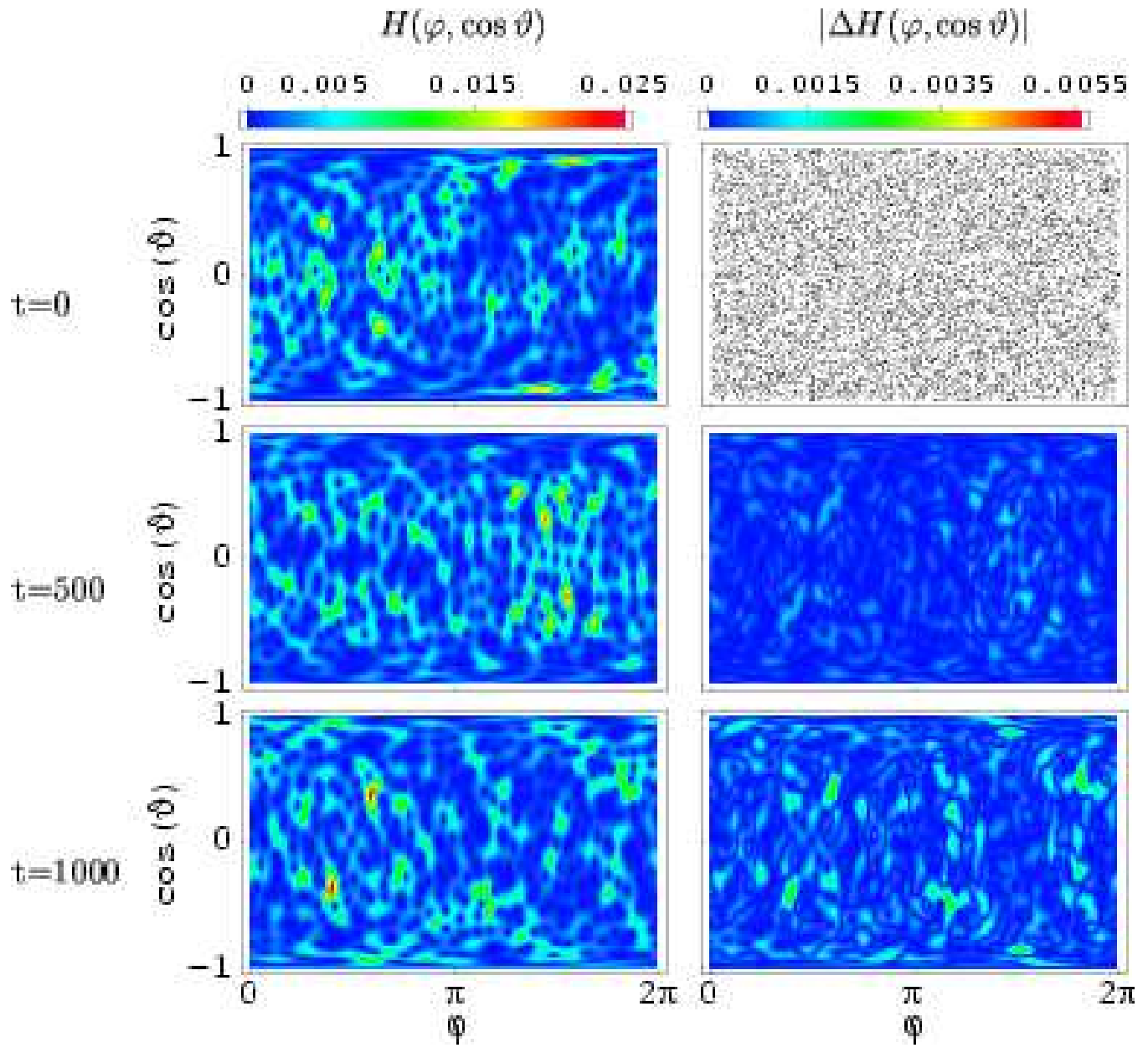

Figure 10. The same as in fig. 9 except for a chaotic (mixing) dynamics, $\alpha=30$. Numerical values of the fidelity are now $|F(500)|=0.997$ and $|F(1000)|=0.988$.

it is unvariant under the permutation of the identical tops. However in our simpleminded numerical experiment reported here we have used the propagator (61) over the full $(2 J+1)^{2}$ dimensional Hilbert space thus making the appropriate average over all symmetry classes.

The classical limit is obtained by $J \rightarrow \infty$ and writing the classical angular momentum vectors in terms of two unit vectors on the sphere $\boldsymbol{r}_{1,2}=\boldsymbol{J}_{1,2} / J$. In component notation we get the following equations of motion

$$
\begin{aligned}
& x_{1,2}^{\prime}=z_{1,2} \\
& y_{1,2}^{\prime}=y_{1,2} \cos \left(\epsilon z_{2,1}\right)+x_{1,2} \sin \left(\epsilon z_{2,1}\right) \\
& z_{1,2}^{\prime}=-x_{1,2} \cos \left(\epsilon x_{2,1}\right)+y_{1,2} \sin \left(\epsilon z_{2,1}\right)
\end{aligned}
$$

We have chosen two regimes, namely non-ergodic (KAM) regime for $\epsilon=1$ where the vast majority of classical orbits are stable, and the mixing regime for $\epsilon=20$ where no significant traces of stable classical orbits were found and where the correlation sum was 


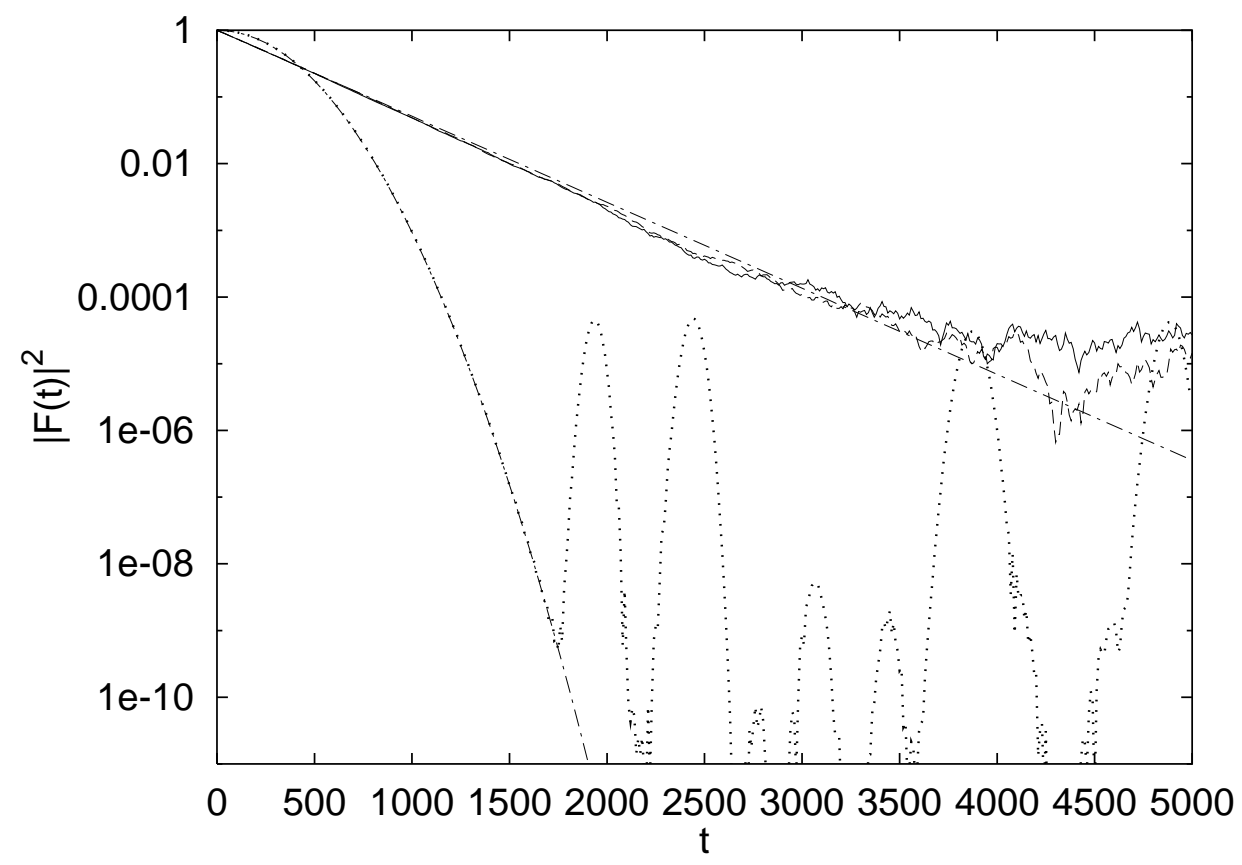

Figure 11. Fidelity for two coupled kicked tops, $\delta=8 \cdot 10^{-4}$ and $J=200$. The upper curves are for $\epsilon=20$ (mixing regime), solid curve for a coherent initial state (65) and dashed curve for a random initial state, and the lower - dotted curve is for $\epsilon=1$ (non-mixing regime) with a coherent initial state (65). The exponential and gaussian chain curves give, respectively, the expected theoretical decays (11) and (27, here decay time is determined by the best fit).

to a very good accuracy given by the first term only

$$
\sigma \approx \frac{1}{2} C(0)=\frac{1}{2 J^{4} \mathcal{N}} \operatorname{tr} J_{1 \mathrm{z}}^{2} J_{2 \mathrm{z}}^{2}=\frac{1}{18}\left(1+\frac{1}{J}\right)^{2} .
$$

Our motivation here was to compare the non-ergodic and mixing fidelity decays for the coherent initial state which is here the dyadic product of $\mathrm{SU}(2)$ coherent states (49)

$$
|\vartheta, \varphi\rangle_{12}=\left|\vartheta_{2}, \varphi_{2}\right\rangle_{2} \otimes\left|\vartheta_{1}, \varphi_{1}\right\rangle_{1}
$$

In fig.11 we show the fidelity decay at $J=200$ and $\delta=8 \cdot 10^{-4}$ in non-ergodic and mixing cases started from the coherent state (65) with $\left(\vartheta_{1}, \varphi_{1}\right)=\left(\vartheta_{2}, \varphi_{2}\right)=\pi(1 / \sqrt{3}, 1 / \sqrt{2})$. We find excellent agreement between the theortical predictions (11) and (27) and the numerics. Note that we are here already in the regime $\delta<\delta_{c}$ where the fidelity decay in ergodic-mixing regime is slower $\tau_{\mathrm{em}}=673$ than in non-ergodic regime $\tau_{\text {ne-coh }}=379$. In the ergodic-mixing regime $(\epsilon=20)$ we show for comparison also the fidelity decay for a random initial state which is (due to ergodicity) almost identical to the case of coherent initial state. We note that overall fidelity decay here is similar as in a one-dimensional case, however, the scaling of various time and perturbation scales on $\hbar=1 / J$ is different as discussed in subsect 2.3. We want to conclude this section with an interesting experimental observation, namely that in the non-ergodic regime the revivals of fidelity (quantum reccurrences) beyond the time scale $t_{*}$ are much less pronounced in $2 d$ than in $1 d$, e.g. compare figs.8 and 11 . 


\section{Classical fidelity}

In this section we wish to contrast the surprising findings on the stability of quantum motion with an application of the general idea (section 2) to a fully classical concept, namely to (unitary) Perron-Frobenius propagator of classical phase space densities. Let us consider a classical dynamical system being given by an invertible measure (volume) preserving map $\phi: \boldsymbol{x} \rightarrow \boldsymbol{\phi}(\boldsymbol{x})$ on a $D(=2 d)$ dimensional phase space $\mathcal{M}$. No additional properties of the map will be assumed except of being at least piece-wise differentiable so that the stability matrix

$$
\begin{aligned}
& \mathbf{M}(\boldsymbol{x})=\frac{\partial \boldsymbol{\phi}(\boldsymbol{x})}{\partial \boldsymbol{x}}, \\
& \mathbf{M}_{t}(\boldsymbol{x})=\frac{\partial \boldsymbol{\phi}^{(t)}(\boldsymbol{x})}{\partial \boldsymbol{x}}=\mathbf{M}\left(\boldsymbol{x}_{t-1}\right) \cdots \mathbf{M}\left(\boldsymbol{x}_{1}\right) \mathbf{M}\left(\boldsymbol{x}_{0}\right), \quad \boldsymbol{x}_{t}=\boldsymbol{\phi}^{(t)}(\boldsymbol{x})
\end{aligned}
$$

exists almost everywhere. Unitary Perron-Frobenius propagator $U_{\mathrm{cl}}$ is defined on a phase space density from $\rho \in L^{2}(\mathcal{M})$ as

$$
\left(U_{\mathrm{cl}} \rho\right)(\boldsymbol{x})=\rho\left(\boldsymbol{\phi}^{(-1)}(\boldsymbol{x})\right) .
$$

The next step is to perturb the map in a most general way by composing it

$$
\phi_{\delta}=\phi \circ g_{\delta}
$$

with a near identity map $\boldsymbol{g}_{\delta}(\boldsymbol{x})$ which is generated by an arbitrary (smooth) vector field $\boldsymbol{a}(\boldsymbol{x})$ thru the $\delta$-flow

$$
\frac{d \boldsymbol{g}_{\delta}}{d \delta}=\boldsymbol{a}\left(\boldsymbol{g}_{\delta}\right), \quad \text { with initial conditions } \quad \boldsymbol{g}_{0}(\boldsymbol{x})=\boldsymbol{x} .
$$

Vector field $\boldsymbol{a}(\boldsymbol{x})$ should be divergence free

$$
\operatorname{div} \boldsymbol{a}(\boldsymbol{x}) \equiv 0
$$

to keep the perturbed map $\phi_{\delta}$ volume preserving (or hamiltonian for symplectic maps). Now we can write the perturbed unitary Perron-Frobenius propagator

$$
\left(U_{\mathrm{cl}, \delta} \rho\right)(\boldsymbol{x})=\rho\left(\boldsymbol{g}_{\delta}^{(-1)}\left(\phi^{(-1)}(\boldsymbol{x})\right)\right)=\left(U_{\mathrm{cl}} \exp (-\delta \boldsymbol{a}(\boldsymbol{x}) \cdot \partial / \partial \boldsymbol{x}) \rho\right)(\boldsymbol{x})
$$

in the standardized general form (1) with the self-adjoint perturbation operator $A_{\mathrm{cl}}$

$$
U_{\mathrm{cl}, \delta}=U_{\mathrm{cl}} \exp \left(-i \delta A_{\mathrm{cl}}\right), \quad A_{\mathrm{cl}}=-i \boldsymbol{a} \cdot \frac{\partial}{\partial \boldsymbol{x}} .
$$

We define the 'classical fidelity' as the inner product between the phase space densities propagated by two slightly different Perron-Frobenius propagators from the same fixed initial density $\rho(\boldsymbol{x})$

$$
F_{\mathrm{cl}}(t)=\int_{\mathcal{M}} d^{D} \boldsymbol{x}\left(U_{\mathrm{cl}, \delta}^{t} \rho\right)(\boldsymbol{x})\left(U_{\mathrm{cl}}^{t} \rho\right)(\boldsymbol{x})=\int_{\mathcal{M}} d^{D} \boldsymbol{x} \rho\left(\phi_{\delta}^{(-t)}(\boldsymbol{x})\right) \rho\left(\boldsymbol{\phi}^{(-t)}(\boldsymbol{x})\right) .
$$

Note that $F_{\mathrm{cl}}(t)$ follows the corresponding quantum fidelity $F(t)(2)$ for very short times

$t \ll t_{\mathrm{E}}$, since the latter can be alternatively written as $F(t)=\int d^{D} \boldsymbol{x} W(\boldsymbol{x}, t) W_{\delta}(\boldsymbol{x}, t)$ in terms of Wigner functions $W(\boldsymbol{x}, t)$ and $W_{\delta}(\boldsymbol{x}, t)$ of the states $U^{t}|\psi\rangle$ and $U_{\delta}^{t}|\psi\rangle$, respectively, corresponding to the Liouville densities $U_{\mathrm{cl}}^{t} \rho$ and $U_{\mathrm{cl}, \delta}^{t} \rho$ in $(74)$ if $\rho(\boldsymbol{x})=$ 
$W(\boldsymbol{x}, t=0) . \quad F_{\mathrm{cl}}(t)$ can be given a probabilistic interpretation, namely if $\rho(\boldsymbol{x})$ is a characteristic function on a set $\mathcal{B} \subset \mathcal{M}, \rho(\boldsymbol{x})=1$ or 0 , for $\boldsymbol{x} \in \mathcal{M}$ or $\boldsymbol{x} \notin \mathcal{M}$, respectively, with the volume $\mathcal{V}=\int_{\mathcal{M}} d^{D} \boldsymbol{x} \rho(\boldsymbol{x})$ then $\mathcal{V}^{-1} F_{\mathrm{cl}}(t)$ is a probability that a perturbed system with the initial condition chosen at random from the set $\mathcal{B}$ is found, after time $t$, in the image of the same set $\mathcal{B}$ propagated for time $t$ with the unperturbed system.

In order to proceed formally along eqs. (44-6) we define and straightforwardly compute the 'classical Heisenberg operators'

$$
A_{\mathrm{cl}, t}:=U_{\mathrm{cl}}^{-t} A_{\mathrm{cl}} U_{\mathrm{cl}}^{t}=-i \boldsymbol{a}_{t}(\boldsymbol{x}) \cdot \frac{\partial}{\partial \boldsymbol{x}}, \quad \boldsymbol{a}_{t}(\boldsymbol{x}):=\mathbf{M}_{t}^{-1}(\boldsymbol{x}) \boldsymbol{a}\left(\phi^{(t)}(\boldsymbol{x})\right) .
$$

Finally, plugging this into $\delta$-expansion (5), observing that the first order always vanishes since $\operatorname{div} \boldsymbol{a}_{t} \equiv 0$, and simplifying the second order by integrating by parts, we obtain

$$
F_{\mathrm{cl}}(t)=1-\frac{\delta^{2}}{2} \int_{\mathcal{M}} d^{D} \boldsymbol{x}\left|\frac{\partial \rho(\boldsymbol{x})}{\partial \boldsymbol{x}} \cdot \sum_{t^{\prime}=0}^{t-1} \mathbf{M}_{t^{\prime}}^{-1}(\boldsymbol{x}) \boldsymbol{a}\left(\phi^{\left(-t^{\prime}\right)}(\boldsymbol{x})\right)\right|^{2}+\mathcal{O}\left(\delta^{3}\right) .
$$

Note that now the RHS does not look like a correlation function at all! The reason for this lies in an essentially different form of the generator of perturbation (73) which in classical case involves phase space derivatives invoking the stability matrix, while in quantum mechanics it is a simple quantization of the observable $\boldsymbol{a}(\boldsymbol{x})$.

Now, observe that the classical linear response formula (76) gives precisely the same result as one intuitively expects based on the stability of individual orbits. Indeed, in the case of chaotic dynamics, the integrand (for sufficiently long times $t^{\prime}$ ) becomes dominated by the leading eigenvalue of the stability matrix $\mathbf{M}_{t}$, namely the largest Lyapunov exponent $\lambda$, and (76) can be written as

$$
F_{\text {cl-cha }}(t)=1-\text { const }_{1} \delta^{2} \exp (2 \lambda t)+\mathcal{O}\left(\delta^{3}\right)
$$

where the unspecified constant depends on the initial distribution $\rho(\boldsymbol{x})$. This means the classical fidelity for chaotic dynamic decays on logarithhmically short time-scale $\sim \log (1 / \delta) / \lambda$. On the other hand, in the case of regular dynamics, all eigenvalues of the stability matrices $\mathbf{M}_{t}$ have modulus 1 so the integrand remains bounded by some constant and the fidelity can be estimated as

$$
F_{\text {cl-reg }}(t)=1-\text { const }_{2} \delta^{2} t^{2}+\mathcal{O}\left(\delta^{3}\right)
$$

Note that the fidelity decay for regular dynamics has the same $\delta$ and $t$ dependence as in the quantum chase (13) with the decay time-scale $\sim \delta^{-1}$.

\section{Summary and discussion}

In this paper we have presented a simple theory on intrinsic decoherence of deterministic (isolated) quantum dynamical systems due to small static perturbations of the evolution operator. The central object of study is the fidelity $F(t)$ of quantum motion computed either with a single (pure) initial state or averaged over an ensemble of initial states described by the statistical density matrix. 
The main result of the paper is a simple linear response (or Kubo-like) formula (6) which relates the fidelity decay to the total sum (or integral) of two point time autocorrelation function of the generator of perturbation. In the limit of infinite Hilbert space dimension we found exponential fidelity decay on a time-scale $\tau_{\mathrm{em}} \propto(\hbar / \delta)^{2}$, for quantum ergodic and mixing systems, whereas for non-ergodic systems we have found much faster decay (in the sufficiently 'quantum' regime where $\delta \ll \hbar$ ) on a time-scale $\tau_{\text {ne }} \propto \hbar / \delta$ for random initial states or $\tau_{\text {ne-coh }} \propto \hbar^{1 / 2} / \delta$ for coherent initial states (minimal uncertainty wavepackets) where the fidelity $F(t)$ is given by a Fourier transformation of the local density of states of the time averaged perturbation operator. A special emphasis was given to the semiclassical theory of fidelity of small but finite values of $\hbar$, where different regimes and the corresponding time and perturbation scales are carefully discussed, and where fidelity decay may asymptotically (as $\hbar \rightarrow 0$ ) be evaluated in terms of classical quantities only. Interestingly, finite size fluctuations of fidelity (for very long times at a finite Hilbert space dimension) have been shown to be given by the inverse participation ratio of the eigenstates of the perturbed evolution operator in the eigenbasis of the unperturbed propagator. The surprising aspects of our relations are mainly due to non-interchangability of the limits $\delta \rightarrow 0$ and $\hbar \rightarrow 0$ as the relevant decay timescales are only functions of the ratio $\delta / \hbar$. Therefore, a different and intuitively expected behaviour, namely faster fidelity decay for mixing than regular dynamics, is obtained in the 'classical' regime where $\hbar \ll \delta$ (or making the limit $\hbar \rightarrow 0$ prior to $\delta \rightarrow 0$ ). A similar, reassuring result has been found by applying our formalism to inspect the analogous classical fidelity for the unitary Perron-Frobenius evolution of volume (area) preserving maps: there the classical fidelity for regular dynamics has been found to decay on a time-scale $\propto \delta^{-2}$, while for a chaotic dynamics, the fidelity decay is governed by a maximal Lyapunov exponent $\lambda$ on a short time-scale $\propto \ln (1 / \delta) / \lambda$.

We note that our findings should be of primary importance for understanding the problem of stability of quantum computation [11] where different regimes and time-scales 25] should now be explained in terms of intrinsic dynamics of a particular quantum algorithm. Actually, our relation between fidelity and correlation decay may prove very useful in design of any new technology which is aimed at manipulating individual quantum states. On the other hand, our results may also shed new light on the relation between decoherence and dynamics [26]. In particular, due to quite special role of coherent initial states on the short Ehrenfest time-scale one may expect the results to be quite different for a random initial state and/or for longer time-scales (see [27]).

\section{Acknowledgements}

We thank T. H. Seligman for very fruitful discussions and for many encouraging and useful remarks. We also acknowledge discussions with G. Usaj, S. Tomsovic and G. Veble. The work has been supported by the Ministry of Education, Science and Sport of Slovenia. 
Stability of quantum motion and correlation decay

\section{References}

[1] Haake F 1991 Quantum signatures of chaos (Berlin: Springer)

[2] Peres A 1995 Quantum Theory: Concepts and Methods (Dordrecht: Kluwer)

[3] Nakamura K 1997 Quantum versus chaos (Dordrecht: Kluwer)

[4] Casati G, Chirikov B V, Guarneri I and Shepelyansky D L 1986 Dynamic stability of quantum chaotic motion in a hydrogen atom Phys. Rev. Lett. 56 2437-40

[5] Peres A 1984 Stability of quantum motion in chaotic and regular systems Phys. Rev. A 30 1610-15; see also the book [2]

[6] Pastawski H M, Levstein P R and Usaj G 1995 Quantum dynamical echoes in the spin-diffusion in mesoscopic systems Phys. Rev. Lett.75 4310-4313; Levstein P R, Usaj G and Pastawski H M 1998 Attenuation of polarization echoes in nuclear magnetic resonance: A study of the emergence of dynamical irreversibility in many-body quantum system J. Chem. Phys. 108 2718-2724.

[7] Nielsen M A and Chuang I L 2000 Quantum computation and quantum information (Cambridge UP)

[8] Shack R, D'Ariano M G and Caves C M 1994 Hypersensitivity to perturbation in the quantum kicked top Phys. Rev. E 50 972-87

[9] Narnhofer H and Thirring W 1989 Mixing property of quantum systems J. Stat. Phys. 57 811-25; Jona-Lasinio G and Presilla C 1996 Chaotic properties of quantum many-body systems in the thermodynamic limit Phys. Rev. Lett. 77 4322-25.

[10] Prosen T 2001 On general relation between quantum ergodicity and fidelity of quantum dynamics preprint quant-ph/0106149, at press in Phys. Rev. E

[11] Prosen T and Žnidarič M 2001 Can quantum chaos enhance stability of quantum computation? at press in J. Phys. A: Math. Gen. 34 L681

[12] Peres A 1984 New conserved quantities and test for regular spectra Phys. Rev. Lett. 53 1711-13

[13] Feingold M and Peres A 1986 Distribution of matrix-elements of chaotic systems Phys. Rev. A 34 591-95; Feingold M, Leitner D M and Piro O 1989 Semiclassical structure of hamiltonians Phys. Rev. A 39 6507-14; Prosen T and Robnik M 1993 Distribution and fluctuation properties of transition probabilities in a system between integrability and chaos J. Phys. A: Math. Gen. 26 L319-26; Prosen T 1994 Statistical properties of matrix elements in a hamilton system between integrability and chaos Ann. Phys. (NY) 235 115-64

[14] Jalabert R A and Pastawski H M 2001 Environment-independent decoherence rate in classically chaotic systems Phys. Rev. Lett. 86 2490-2493

[15] Cucchietti F M, Pastawski H M and Wisniacki D A 2001 Sensitivity of the Loschmidt Echo as a monitor of dynamical Quantum Chaos preprint cond-mat/0102135

[16] Jacquod Ph, Silvestrov P G, Beenakker C W J 2001 Golden rule decay versus Lyapunov decay of the quantum Loschmidt echo Phys. Rev. E 64055203

[17] Tomsovic S and Cerruti N R 2001 Sensitivity of Wave Field Evolution and Manifold Stability in Chaotic Systems preprint nlin.CD/0108016

[18] Alicki R, Makowiec D and Miklaszewski W 1996 Quantum Chaos in Terms of Entropy for a Periodically Kicked Top Phys. Rev. Lett. 77 838-41

[19] Haake F, Kuś M and Scharf R 1987 Z. Phys. B 65 381; see also the book [1]

[20] Fox R F and Elston T C 1994 Chaos and a quantum-classical correspondence in the kicked top Phys. Rev. E 50 2553-63

[21] Casati G, Chirikov B V and Zhirov O V 1997 Existence of a long time scale in quantum chaos Phys. Rev. E 55 7757-8

[22] Breslin J K and Milburn G J 1999 Sensitivity to measurement errors in quantum kicked top Phys. Rev. A 59 1781-87

[23] Haake F 2000 Can the kicked top be realized? J. Mod. Optic. 47 2883-90

[24] Miller P A and Sarkar S 1999 Signatures of chaos in the entanglement of two coupled kicked tops Phys. Rev. E 60 1542-50

[25] Song P H and Shepelyansky D L 2001 Quantum Computing of Quantum Chaos and Imperfection 
Effects Phys. Rev. Lett. 86 2162-65; Georgeot B and Shepelyansky D L 2001 Stable quantum computation of unstable classical chaos Phys. Rev. Lett. 86 5393-96 (also quant-ph/0101004) and Efficient quantum computing insensitive to phase errors preprint quant-ph/0102082

[26] Zurek W H and Paz J P 1995 Quantum chaos: a decoherent definition Physica 83D 300-8

[27] Gorin T and Seligman T H 2001 Decoherence in chaotic and integrable systems: A random matrix approach preprint $\mathrm{nlin. \textrm {CD } / 0 1 0 1 0 1 8}$ 